\title{
Atomistically determined phase-field modeling of dislocation dissociation, stacking fault formation, dislocation slip, and reactions in fcc systems
}

\author{
Jaber Rezaei Mianroodi \\ Material Mechanics, RWTH Aachen University, Schinkelstr. 2, 52062 Aachen, Germany \\ Bob Svendsen \\ Material Mechanics, RWTH Aachen University, Schinkelstr. 2, 52062 Aachen, Germany \\ Microstructure Physics and Alloy Design, Max-Planck Institute for Iron Research, Max-Planck Str. 1, 40237 \\ Düsseldorf, Germany
}

\begin{abstract}
The purpose of the current work is the development of a phase field model for dislocation dissociation, slip and stacking fault formation in single crystals amenable to determination via atomistic or $a b$ initio methods in the spirit of computational material design. The current approach is based in particular on periodic microelasticity (Wang et al., 2001; Bulatov and Cai, 2006; Wang and Li, 2010) to model the strongly non-local elastic interaction of dislocation lines via their (residual) strain fields. These strain fields depend in turn on phase fields which are used to parameterize the energy stored in dislocation lines and stacking faults. This energy storage is modeled here with the help of the "interface" energy concept and model of Cahn and Hilliard (1958) (see also Allen and Cahn, 1979; Wang and Li, 2010). In particular, the "homogeneous" part of this energy is related to the "rigid" (i.e., purely translational) part of the displacement of atoms across the slip plane, while the "gradient" part accounts for energy storage in those regions near the slip plane where atomic displacements deviate from being rigid, e.g., in the dislocation core. Via the attendant global energy scaling, the interface energy model facilitates an atomistic determination of the entire phase field energy as an optimal approximation of the (exact) atomistic energy; no adjustable parameters remain. For simplicity, an interatomic potential and molecular statics are employed for this purpose here; alternatively, ab initio (i.e., DFT-based) methods can be used. To illustrate the current approach, it is applied to determine the phase field free energy for fcc aluminum and copper. The identified models are then applied to modeling of dislocation dissociation, stacking fault formation, glide and dislocation reactions in these materials. As well, the tensile loading of a dislocation loop is considered. In the process, the current thermodynamic picture is compared with the classical mechanical one as based on the Peach-Köhler force.
\end{abstract}

Keywords: phase field, molecular statics, stacking fault, stacking fault energy, dislocation core, atomistically determined 


\section{Introduction}

As attested to by theory (e.g., Hirth and Lothe, 1982), atomistic simulation (e.g. Swygenhoven et al., 2006), and experiment (e.g., HRTEM), the energetically favored state of a dislocation line or loop in close-packed metals is its dissociated state, consisting of leading and trailing partial dislocations bounding, and separated by, a stacking fault (SF). The details of this configuration are dynamic in nature. In particular, the size of the stacking fault depends on the force balance between the long-range stress-field-based repulsion of the leading and trailing partials, the shortrange attraction resulting from the energy cost of increasing the SF size, and any external loading forces. On the material side, then, the energy needed to create an SF in a given material, i.e., the stacking fault energy (SFE), plays a predominant role. In particular, the relation of the SFE to the level of loading is decisive in determining whether or not in a given material SFs shrink to their equilibrium size or continue to grow dynamically during loading. This in turn may influence a number of other dislocation processes such as core reactions, dislocation-based structural transformation (e.g., martensite formation), dislocation-based twinning, cross slip, and network formation (e.g., Hirth and Lothe, 1982).

In order to model the energetics and kinetics of such dissociation-based dislocation processes, at least two approaches have been developed in the literature (for a comprehensive review, see Wang and Li, 2010). The first approach (e.g., Xiang et al., 2008; Hunter et al., 2011, 2013; Shen et al., 2014) is based on various generalizations of the Peierls-Nabarro (PN) dislocation model (e.g., Hirth and Lothe, 1982; Mura, 1987; Wang and Li, 2010). The second approach (e.g., Shen and Wang, 2003, 2004), to which the current work belongs, is based on the "interface" energy model and concept of Cahn and Hilliard (1958); for simplicity, it will be referred to here as the phase field ${ }^{1}$ (PF) model. In both approaches, the free energy is modeled on contributions from lattice strain (elastic) and from lattice resistance to dislocation motion via homogeneous ("rigid") atomic displacement across the slip plane; in particular, the latter is associated with the (generalized) SFE (e.g., Vitek, 1968). The PF model accounts in addition for a "gradient" contribution ("correction") to energy storage due to non-homogeneous atomic displacement across the slip plane, e.g., in the dislocation core. In both approaches, the elastic energy is based on (or at least consistent with) the Khachaturyan-Shalatov approach (e.g., Khachaturyan, 1983; Wang et al., 2001) for periodic defective systems in which defects are modeled via their (residual) strain fields. Basically following Schoeck (2001, 2003, 2005), Shen and Wang (2004) modeled the SFE via a truncated Fourier series ${ }^{2}$ parameterized by the phase fields. In particular, they fit this to DFT data on the SFE for $\mathrm{Al}$ and $\mathrm{Pd}$. The only undetermined material property in their model is the gradient energy coefficient. In contrast, in the current work, the SFE and gradient energy are determined simultaneously and completely in a material-dependent fashion using atomistic energy and core morphology (e.g., size) data; no adjustable parameters remain. To this end, the global energy scaling approach of Cahn and Hilliard (1958) is exploited in this work to connect (discrete) atomistic and (continuum) PF modeling of dislocation processes. For simplicity, this is based here on an

\footnotetext{
${ }^{1}$ This terminology is not uniform in the literature; for example, Hunter et al. $(2011,2013)$ refer to their model as a phase field model.

${ }^{2}$ Such a truncated Fourier-series representation of this energy is also employed by Xiang et al. (2008), Hunter et al. (2011, 2013) and Shen et al. (2014).
} 
atomic potential and molecular statics; alternatively, $a b$ initio (i.e., DFT-based) methods can be used.

The paper begins in Section 2 with a synopsis of basic model relations and in particular the PF free energy model. This is followed in Section 3 by the reformulation of this model in a form amenable to atomistic or ab initio determination as discussed above via global energy scaling. To demonstrate its use, the resulting approach is applied to the atomistic determination of the PF free energy for $\mathrm{Al}$ and $\mathrm{Cu}$ in Section 4. For simplicity, this is carried out here with the help of an atomic potential in the context of molecular statics (MS); as mentioned above, ab initio DFT data can also be employed. After verification, these atomistically determined PF models for $\mathrm{Al}$ and $\mathrm{Cu}$ are then applied in Section 5 to the modeling of dislocation dissociation, SF formation, dislocation slip, and reactions in these materials the context of the tensile loading of a dislocation loop. In the process, connection is made with the dislocation (line) mechanics as based on Peach-Köhler force (PKF). The paper ends in Section 6 with a summary and discussion. For completeness, selected details of the algorithm employed for the numerical solution of the current model in the simulations are provided in Appendix A. As well, details concerning the relation between thermodynamic driving forces and the PKF are summarized in Appendix B. For simplicity, attention is restricted to infinite periodic fcc systems and purely bulk behavior in this work.

Before we begin, a word on notation. In this work, (three-dimensional) Euclidean vectors are represented by lower-case bold italic characters $\boldsymbol{a}, \boldsymbol{b}, \ldots$, and second-order Euclidean tensors by upper-case bold italic characters $\boldsymbol{A}, \boldsymbol{B}$, etc.; in particular, $\boldsymbol{I}$ is the second-order identity tensor. Third- and fourth-order Euclidean tensors in this work are denoted by upper-case slanted sans-serif characters $A, B$, and so on. The notation $\boldsymbol{a} \cdot \boldsymbol{b}, \boldsymbol{A} \cdot \boldsymbol{B}$, and so on, is used for the scalar product of the corresponding tensors. Let $(\boldsymbol{a} \otimes \boldsymbol{b}) \boldsymbol{c}:=(\boldsymbol{b} \cdot \boldsymbol{c}) \boldsymbol{a}$ define the tensor or dyadic product of $\boldsymbol{a}, \boldsymbol{b}$ as usual. Further, let sym $\boldsymbol{A}:=\frac{1}{2}\left(\boldsymbol{A}+\boldsymbol{A}^{\mathrm{T}}\right)$ represent the symmetric part of any second-order tensor $\boldsymbol{A}$. Additional notation will be introduced as needed in what follows.

\section{Basic model relations}

For simplicity, the current formulation is restricted to single-constituent materials, isothermal conditions, quasi-static loading, and geometric linearity. Again for simplicity, attention is restricted here to single crystals and purely bulk behavior. Under these conditions, consider an inhomogeneous single crystal in which the inhomogeneity results from interacting material defects resulting in residual strain and stress states. Of particular interest in this work is the case of dislocations. Let $\boldsymbol{u}$ represent the displacement field, $\boldsymbol{H}=\nabla \boldsymbol{u}$ the distortion field, and $\boldsymbol{E}=\operatorname{sym} \nabla \boldsymbol{u}$ the strain field. Following many authors (e.g., Kröner, 1959; Eshelby, 1970; Khachaturyan, 1983; Mura, 1987; Wang et al., 2001; Li and Wang, 2008), let

$$
\psi_{\mathrm{E}}=\frac{1}{2}\left(\boldsymbol{E}-\boldsymbol{E}_{\mathrm{R}}\right) \cdot C_{\mathrm{E}}\left(\boldsymbol{E}-\boldsymbol{E}_{\mathrm{R}}\right)
$$

represent the density of stored energy in the single crystal due to lattice deformation determining in particular the corresponding energetic stress

$$
\boldsymbol{T}=\partial_{\boldsymbol{E}} \psi_{\mathrm{E}}=C_{\mathrm{E}}\left(\boldsymbol{E}-\boldsymbol{E}_{\mathrm{R}}\right) .
$$


Here, $\boldsymbol{C}_{\mathrm{E}}$ is the (constant isothermal) elastic stiffness, and $\boldsymbol{E}_{\mathrm{R}}=\operatorname{sym} \boldsymbol{H}_{\mathrm{R}}$ the residual strain due to defects. Restricting attention to fcc glide dislocations and dislocation slip (i.e., no climb), the corresponding distortion field

$$
\boldsymbol{H}_{\mathrm{R}}(\boldsymbol{\phi})=\sum_{a=1}^{p} \phi_{a} \boldsymbol{H}_{a}
$$

is modeled as a sum of $p$ (constant isothermal) elementary contributions

$$
\boldsymbol{H}_{a}=\boldsymbol{b}_{a} \otimes \boldsymbol{n}_{a} / d_{a}=\gamma_{a} \boldsymbol{s}_{a} \otimes \boldsymbol{n}_{a},
$$

each weighted by corresponding phase field $\phi_{a}$. Here, $\gamma_{a}:=b_{a} / d_{a}, b_{a}:=\left|\boldsymbol{b}_{a}\right|, \boldsymbol{s}_{a}:=\boldsymbol{b}_{a} / b_{a}, \boldsymbol{b}_{a}$ is the Burgers vector, $d_{a}=d_{111}=a_{0} / \sqrt{3}$ is the $\{111\}$ interplanar spacing, $a_{0}$ is the lattice constant, and $\boldsymbol{n}_{a}$ is the slip plane unit normal. Each $\phi_{a}$ models the transition between different states of slip in the energy landscape of the single crystal (more on this below). These fields are modeled such that $\boldsymbol{H}_{\mathrm{R}}$ vanishes everywhere in the material except on slipped surfaces (i.e., has "support" only on such surfaces).

Following Schoeck (2001) and Bulatov and Cai (2006, Chapter 11), the current approach is based on two $\phi_{a}$ per plane. Besides being computationally more efficient than three such fields per plane (as done for example by Shen and Wang, 2004), this facilitates the model investigation of processes based on perfect $\langle 110\rangle$ or (Shockley) partial $\langle 112\rangle$ dislocations alone. As an example of such PFs, consider those $\phi_{1}$ and $\phi_{2}$ for the (111) plane as shown in Figure 1. Given two PFs per

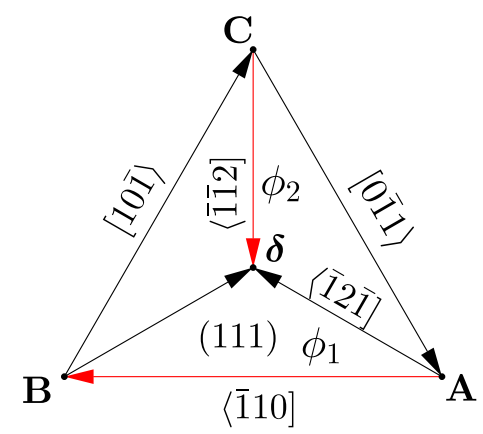

Figure 1: Shear directions (red arrows) on the (111) plane associated with the PFs $\phi_{1}$ and $\phi_{2}$ depicted on the (111) side of the Thompson tetrahedron. As shown, $\phi_{1}$ is associated with simple shear strain $\boldsymbol{E}_{1}=\gamma_{1} \operatorname{sym}\left(\boldsymbol{s}_{1} \otimes \boldsymbol{n}_{1}\right)$ for perfect slip $\boldsymbol{s}_{1} \equiv[\overline{1} 10] / \sqrt{2}$, and $\phi_{2}$ with that $\boldsymbol{E}_{2}=\gamma_{2} \operatorname{sym}\left(\boldsymbol{s}_{2} \otimes\right.$ $\boldsymbol{n}_{2}$ ) for partial slip $\boldsymbol{s}_{2} \equiv[\overline{1} \overline{1} 2] / \sqrt{6}$, with $\boldsymbol{n}_{1}=\boldsymbol{n}_{2} \equiv[111] / \sqrt{3}$. In addition, note that $\gamma_{1}=b_{1} / d_{111}=$ $\sqrt{3} / \sqrt{2}$ and $\gamma_{2}=b_{2} / d_{111}=1 / \sqrt{2}$ via $b_{1}=a_{0} / \sqrt{2}, b_{2}=a_{0} / \sqrt{6}$ and $d_{111}=a_{0} / \sqrt{3}$.

\{111\} plane, we have $p=8$ PFs for general octahedral slip in an fcc single crystal. In particular, both straight dislocation lines and dislocation loops (e.g., Eshelby, 1961; Bulatov and Cai, 2006, Chapter 11) can be modeled in this fashion.

Under quasi-static loading conditions, the timescale for "displacive" deformation of the lattice is assumed to be much shorter than that for "diffusive" deformation due to the motion of 
defect systems. As such, the lattice is able to react "instantaneously" to such motion and maintain mechanical equilibrium

$$
\operatorname{div} \boldsymbol{T}=\mathbf{0} .
$$

Idealizing dislocation motion as "overdamped" and relaxational in nature, the evolution or dynamics of each $\phi_{a}$ is modeled via the (time-dependent) Ginzburg-Landau relation

$$
\dot{\phi}_{a}=-m_{0} \delta_{\phi_{a}} \psi=m_{0}\left(\operatorname{div} \partial_{\nabla \phi_{a}} \psi-\partial_{\phi_{a}} \psi\right)
$$

subject to no-flux (periodic) boundary conditions

$$
\partial_{\nabla \phi_{a}} \psi \cdot \boldsymbol{n}=0
$$

for each system $a$. Non-negative mobility $m_{0} \geqslant 0$ (assumed constant and equal for all $a=1, \ldots, 8$ for simplicity) insures non-negative dissipation and gradient flow of the system toward thermodynamic equilbrium.

The basic model relations are completed by the form

$$
\psi(\nabla \boldsymbol{u}, \boldsymbol{\phi}, \nabla \boldsymbol{\phi})=\psi_{\mathrm{E}}(\nabla \boldsymbol{u}, \boldsymbol{\phi})+\psi_{\mathrm{C}}(\boldsymbol{\phi}, \nabla \boldsymbol{\phi})
$$

for the total free energy density $\psi$ of the single crystal, with $\psi_{\mathrm{C}}$ its "chemical" part, and $\boldsymbol{\phi}:=$ $\left(\phi_{1}, \ldots, \phi_{p}\right)$. In particular, in the context of the local gradient, or interface, approximation of Cahn and Hilliard (1958), this latter is modeled as the sum

$$
\psi_{\mathrm{C}}(\boldsymbol{\phi}, \nabla \boldsymbol{\phi})=\psi_{\mathrm{H}}(\boldsymbol{\phi})+\psi_{\mathrm{G}}(\nabla \boldsymbol{\phi})
$$

of "homogeneous" $\psi_{\mathrm{H}}$ and "gradient" $\psi_{\mathrm{G}}$ parts. As already mentioned above, the current choice of PFs as depicted in Figure 1 simplifies the modeling of $\langle 110\rangle$ perfect slip and $\langle 112\rangle$ partial slip on $\{111\}$ planes. From a physical point of view, perfect dislocation slip (on multiple planes) results in no atomic restacking and so preserves lattice orientation. On the other hand, partial dislocation slip is accompanied by SF formation; such slip on successive $\{111\}$ planes results in atomic restacking and lattice reorientation or even transformation (e.g., Hirth and Lothe, 1982; Christian and Mahajan, 1995). In this way, $\psi_{\mathrm{C}}$, and in particular $\psi_{\mathrm{H}}$, becomes associated with the energy needed to create, or stored in, stacking faults, i.e., the (generalized) SFE (e.g., Vitek, 1968). Indeed, as discussed above, $\psi_{\mathrm{H}}$ is associated with the homogeneous ("rigid") displacement of atoms across $\{111\}$ planes due to dislocation slip. To account for the fact that such atomic displacement is in fact inhomogeneous, especially in the region around the dislocation core, $\psi$ depends as well on $\psi_{\mathrm{G}}$. More generally, $\psi_{\mathrm{G}}$ also accounts for the influence of properties such as bonding type (Schoeck, 2005, 2006; Wang and Li, 2010). Since the current work is restricted to dislocation slip, the dependence of $\psi_{\mathrm{G}}$ on $\nabla \phi$ at the single crystal level is "parameterized" by the Nye measure curl $\boldsymbol{H}_{\mathrm{R}}$, which projects $\nabla \boldsymbol{\phi}$ onto the slip systems. Indeed, from (3) and (4),

$$
\operatorname{curl} \boldsymbol{H}_{\mathrm{R}}=\sum_{a=1}^{p} \gamma_{a} \boldsymbol{s}_{a} \otimes \nabla \phi_{a} \times \boldsymbol{n}_{a}=\sum_{a=1}^{p} \gamma_{a}\left\{\left(\nabla_{\boldsymbol{t}_{a}} \phi_{a}\right) \boldsymbol{s}_{a} \otimes \boldsymbol{s}_{a}-\left(\nabla_{\boldsymbol{s}_{a}} \phi_{a}\right) \boldsymbol{s}_{a} \otimes \boldsymbol{t}_{a}\right\}
$$


depends only on the slip plane "components" $\nabla_{s_{a}} \phi_{a}=\nabla \phi_{a} \cdot \boldsymbol{s}_{a}$ and $\nabla_{t_{a}} \phi_{a}=\nabla \phi_{a} \cdot \boldsymbol{t}_{a}$ of $\nabla \phi_{a}$. Here, $\boldsymbol{t}_{a}=\boldsymbol{n}_{a} \times \boldsymbol{s}_{a}$ and $\left(\boldsymbol{s}_{a}, \boldsymbol{t}_{a}, \boldsymbol{n}_{a}\right)$ an orthogonal right-hand system. For example, $\boldsymbol{t}_{1} \equiv[111] / \sqrt{3} \times$ $[\overline{1} 10] / \sqrt{2}=[\overline{1} \overline{1} 2] / \sqrt{6} \equiv s_{2}$ and $t_{2} \equiv[111] / \sqrt{3} \times[\overline{1} \overline{1} 2] / \sqrt{6}=[1 \overline{1} 0] / \sqrt{2} \equiv-s_{1}$ for the (111) glide plane as discussed above in the context of Figure 1. On this basis, $\psi_{\mathrm{G}}$ is modeled here for simplicity by the ubiquitous quadratic form

$$
\psi_{\mathrm{G}}(\nabla \boldsymbol{\phi})=\psi_{\mathrm{G} 0}\left|\operatorname{curl} \boldsymbol{H}_{\mathrm{R}}\right|^{2}=\psi_{\mathrm{G} 0} \sum_{a, b=1}^{p} \nabla \phi_{a} \cdot \boldsymbol{N}_{a b}^{g} \nabla \phi_{b},
$$

with

$$
\boldsymbol{N}_{a b}^{g}:=\gamma_{a} \gamma_{b}\left(\boldsymbol{s}_{a} \cdot \boldsymbol{s}_{b}\right)\left\{\left(\boldsymbol{n}_{a} \cdot \boldsymbol{n}_{b}\right) \boldsymbol{I}-\boldsymbol{n}_{b} \otimes \boldsymbol{n}_{a}\right\} .
$$

Note that the scaling factor $\psi_{\mathrm{G} 0}$ has unit of energy per unit length. If interactions among distinct slip systems $(a \neq b)$ are neglected, (11) reduces to the relation considered for example by Bulatov and Cai (2006, Equation (11.34)).

Since we are interested in purely bulk behavior of fcc single crystals in this work, attention is restricted to periodic fields and systems, and Fourier transform methods are applicable for solution of initial boundary value problems based on (5)-(7) as usual (e.g., Khachaturyan, 1983; Mura, 1987; Suquet, 1997; Nemat-Nasser and Hori, 1999; Li and Wang, 2008). Both general, algorithmic and numerical details in this regard are summarized for completeness in Appendix A.

\section{Model formulation based on energy scaling}

As discussed in the introduction, the determination of the PF free energy with the help of atomistic methods in this work is based on adaptation of the interface energy and scaling approach of Cahn and Hilliard (1958) to the current case. To this end, a characteristic process is chosen which is amenable to both atomistic (here molecular static; MS) and PF modeling. To this end, consider the dissociation

$$
\begin{aligned}
& \mathrm{AB} \longrightarrow \mathrm{A} \delta+\delta \mathrm{B} \\
& \frac{a_{0}}{2}[\overline{1} 10] \longrightarrow \frac{a_{0}}{6}[\overline{1} 2 \overline{1}]+\frac{a_{0}}{6}[\overline{2} 11]
\end{aligned}
$$

of a single perfect screw dislocation in the (111) plane with Burgers vector $\mathbf{A B}=\frac{a_{0}}{2}[\overline{1} 10], a_{0}$ being the lattice constant. In terms of $\left(\phi_{1}, \phi_{2}\right)$ in Figure 1 , note that $\mathbf{A B} \equiv(1,0), \mathbf{A} \boldsymbol{\delta} \equiv\left(\frac{1}{2},-\frac{1}{2}\right)$, and $\boldsymbol{\delta} \mathbf{B} \equiv\left(\frac{1}{2}, \frac{1}{2}\right)$. For the formulation to follow, it proves useful to work with the related Cartesian basis system

$$
\boldsymbol{i}_{x} \equiv[\overline{1} \overline{1} 2] / \sqrt{6}, \quad \boldsymbol{i}_{y} \equiv[111] / \sqrt{3}, \quad \boldsymbol{i}_{z} \equiv[\overline{1} 10] / \sqrt{2} .
$$

Being a screw dislocation, $\mathbf{A B}$ then has line direction $\boldsymbol{i}_{z}$. Further, $\mathbf{A} \boldsymbol{\delta}=-\frac{1}{2} b_{112} \boldsymbol{i}_{x}+\frac{1}{2} b_{110} \boldsymbol{i}_{z}$ and $\boldsymbol{\delta} \mathbf{B}=\frac{1}{2} b_{112} \boldsymbol{i}_{x}+\frac{1}{2} b_{110} \boldsymbol{i}_{z}$ then hold relative to (14), with $b_{110}=a_{0} / \sqrt{2}$ and $b_{112}=a_{0} / \sqrt{6}$. Likewise, $\boldsymbol{n}_{1}=\boldsymbol{n}_{2}=\boldsymbol{i}_{y}, \boldsymbol{s}_{1}=\boldsymbol{i}_{z}, \boldsymbol{s}_{2}=\boldsymbol{i}_{x}, \boldsymbol{t}_{1}=\boldsymbol{s}_{2}=\boldsymbol{i}_{x}$, and $\boldsymbol{t}_{2}=-\boldsymbol{s}_{1}=-\boldsymbol{i}_{z}$. In addition, $\phi_{2}$ is then associated with $\boldsymbol{i}_{x}$, and $\phi_{1}$ with $\boldsymbol{i}_{z}$. So set $\phi_{x}=\phi_{2}$ and $\phi_{z}=\phi_{1}$ in what follows. 
Adapting now the approach of Cahn and Hilliard (1958) to the current case of a single (111) slip plane, the relation

$$
\begin{aligned}
\psi_{\mathrm{EH}}\left(\phi_{x}, \phi_{z}\right)-\psi_{\mathrm{EH}}(0,0) & =\Delta \psi_{\mathrm{EH}}\left(\phi_{x}, \phi_{z}\right) \\
& =\psi_{\mathrm{G}}\left(\nabla \phi_{x}, \nabla \phi_{z}\right) \\
& =\frac{1}{2} \psi_{\mathrm{G} 0}\left|\nabla_{i_{x}} \phi_{x}\right|^{2}+\frac{3}{2} \psi_{\mathrm{G} 0}\left|\nabla_{i_{x}} \phi_{z}\right|^{2}
\end{aligned}
$$

is obtained in equilibrium between the effective homogeneous ${ }^{3}$

$$
\psi_{\mathrm{EH}}\left(\phi_{x}, \phi_{z}\right):=\psi_{\mathrm{E}}\left(\phi_{x}, \phi_{z}\right)+\psi_{\mathrm{H}}\left(\phi_{x}, \phi_{z}\right),
$$

and gradient $\psi_{\mathrm{G}}\left(\nabla \phi_{x}, \nabla \phi_{z}\right)$, parts of $\psi$ from (8) reduced to the current case of a single (111) slip plane. In particular, (15) follows from solution of the Ginzburg-Landau relation (6) in equilbrium $\left(\dot{\phi}_{a}=0\right)$ for $a=x, z$, taking into account the fact that $\nabla_{i_{z}} \phi_{x}$ and $\nabla_{i_{z}} \phi_{z}$ along the line direction $\boldsymbol{i}_{z}$ vanish for straight lines. Given (1) and (3), note that $\psi_{\mathrm{E}}(0,0)=\frac{1}{2} \boldsymbol{E} \cdot \boldsymbol{C}_{\mathrm{E}} \boldsymbol{E}$ is the free energy density of a crystal without defects. Since $\psi_{\mathrm{H}}(0,0)=0$ can be assumed without loss of physical generality, $\psi_{\mathrm{EH}}(0,0)=\psi_{\mathrm{E}}(0,0)=\frac{1}{2} \boldsymbol{E} \cdot \boldsymbol{C}_{\mathrm{E}} \boldsymbol{E}$ holds as well. To proceed further, it is useful to work with the transformations

$$
\boldsymbol{i}=\frac{1}{\sqrt{2}} \boldsymbol{i}_{x}+\frac{1}{\sqrt{2}} \boldsymbol{i}_{z}, \quad \phi \boldsymbol{i}=\phi_{x} \boldsymbol{i}_{x}+\phi_{z} \boldsymbol{i}_{z}, \quad \phi_{x}(\phi)=\frac{1}{\sqrt{2}} \phi, \quad \phi_{z}(\phi)=\frac{1}{\sqrt{2}} \phi .
$$

From these follow the reduced form

$$
\psi_{\mathrm{H} 0} \Delta f_{\mathrm{EH}}(\phi):=\Delta \psi_{\mathrm{EH}}\left(\phi_{x}(\phi), \phi_{z}(\phi)\right)=\psi_{\mathrm{G} 0}\left|\partial_{x} \phi\right|^{2}
$$

of (15) with $\partial_{x} \phi=\nabla_{\boldsymbol{i}_{x}} \phi$. Here, $\Delta f_{\mathrm{EH}}(\phi)$ represents the functional form of $\Delta \psi_{\mathrm{EH}}\left(\phi_{x}(\phi), \phi_{z}(\phi)\right)$, and $\psi_{\mathrm{H} 0}$ is an energy scaling parameter. In turn, (18) implies

$$
\left|\partial_{x} \phi\right|=\sqrt{\frac{\psi_{\mathrm{H} 0}}{\psi_{\mathrm{G} 0}}} \sqrt{\Delta f_{\mathrm{EH}}(\phi)}
$$

for the (magnitude of the) equilibrium PF gradient.

Consider next a region $P$ of the single crystal containing the slip plane in the form of a retangular parallelepiped infinitely long in the $\boldsymbol{i}_{x}$ direction and having finite constant cross section perpendicular to $x$ with area $a_{P}$. Let

$$
g_{0}:=\int_{-\infty}^{\infty} \psi d x=\frac{1}{a_{P}} \int_{P} \psi d v
$$

represent the stored energy per unit cross sectional area. In equilibrium, this takes the form

$$
g_{0}=\kappa_{\mathrm{H} 0} \sqrt{\psi_{\mathrm{H} 0} \psi_{\mathrm{G} 0}}, \quad \kappa_{\mathrm{H} 0}:=\int_{\phi(-\infty)}^{\phi(+\infty)} \frac{f_{\mathrm{EH}}(\phi)+\Delta f_{\mathrm{EH}}(\phi)}{\sqrt{\Delta f_{\mathrm{EH}}(\phi)}} d \phi
$$

\footnotetext{
${ }^{3}$ For simplicity, $\boldsymbol{E}$ is left out of the notation here.
} 
via (18) and (19). Again following Cahn and Hilliard (1958), consider next the model relation

$$
l_{0}:=\frac{\phi(+\infty)-\phi(-\infty)}{\left|\partial_{x} \phi(0)\right|}
$$

for the "width" of the interface region. In the current context, the interface is identified with the dislocation core, and $l_{0}$ with the core size. Substitution of $\partial_{x} \phi(0)$ from (19) (assumed positive) into (22) yields

$$
l_{0}=\kappa_{\mathrm{G} 0} \sqrt{\frac{\psi_{\mathrm{G} 0}}{\psi_{\mathrm{H} 0}}}, \quad \kappa_{\mathrm{G} 0}:=\frac{\phi(+\infty)-\phi(-\infty)}{\sqrt{\Delta f_{\mathrm{EH}}(\phi(0))}} .
$$

Lastly, combination of (21) and (23) results in the forms

$$
\psi_{\mathrm{H} 0}=\frac{\kappa_{\mathrm{G} 0}}{\kappa_{\mathrm{H} 0}} \frac{g_{0}}{l_{0}}, \quad \psi_{\mathrm{G} 0}=\frac{1}{\kappa_{\mathrm{G} 0} \kappa_{\mathrm{H} 0}} g_{0} l_{0},
$$

for the material parameters scaling $\psi_{\mathrm{EH}}=\psi_{\mathrm{E}}+\psi_{\mathrm{H}}$ and $\psi_{\mathrm{G}}$, respectively, in $\psi$ from (8) and (9). As is well-known (e.g., Bulatov and Cai, 2006), the mobility $m_{0}$ can be determined as well atomistically via molecular dynamics (MD). Since the focus of the current work is on energetics, however, this is not done here. Note that the corresponding scaled form $\dot{\phi}_{a}=-m_{0} \psi_{\mathrm{H} 0} \delta_{\phi_{a}} \psi / \psi_{\mathrm{H} 0}$ of (6) implies that the mobility $m_{0}$ is scaled by $\psi_{\mathrm{H} 0}$ as well.

In the next section, the elastic stiffness $C_{\mathrm{E}}$ and so $\psi_{\mathrm{E}}$, as well as $\psi_{\mathrm{H}}, g_{0}$, and $l_{0}$, are all determined from an atomic potential via MS. In particular, the SFE contribution $\psi_{\mathrm{H}}$ is obtained from $\psi_{\mathrm{H}}^{\langle 111\rangle}\left(\phi_{1}, \phi_{2}\right)$ and material symmetry. These determine in turn $\psi_{\mathrm{EH}}=\psi_{\mathrm{E}}+\psi_{\mathrm{H}}$, and so $\kappa_{\mathrm{H} 0}$ from (21). Further, since $\phi_{x, z}(-\infty)=0$ and $\phi_{x, z}(+\infty)=1 / 2$, note that $\phi(-\infty)=0$ and $\phi(+\infty)=1 / \sqrt{2}$ via (17) are known for the case of dissociation considered above. Then $\kappa_{\mathrm{G} 0}$ follows as well from (23). Given all of these, $\psi_{\mathrm{H} 0}$ and $\psi_{\mathrm{G} 0}$ follow from (24), and $\psi$ is completely determined.

\section{Atomistic identification of the PF free energy model}

For comparison, the fcc materials $\mathrm{Al}$ and $\mathrm{Cu}$ are considered here. Besides different lattice and elastic properties, these are characterized by having different SFEs. Calculation of all energetic properties of the PF model discussed in the previous sections for these materials is based here on the corresponding embedded atom potentials from Mishin et al. (1999) for Al and Mishin et al. (2001) for $\mathrm{Cu}$, both at zero temperature. These potentials are widely used and in close agreement with DFT and experimental results (e.g., Zimmerman et al., 2000) for the material properties of interest in this work.

\subsection{Basic properties \& $\psi_{\mathrm{E}}$}

Values for the lattice constant and elastic stiffnesses of $\mathrm{Cu}$ and $\mathrm{Al}$ are summarized in Table 1. Besides $C_{\mathrm{E}}$, the material property values in Table 1 determine the effective fcc shear modulus $\mu_{\mathrm{fcc}}=\left(C_{11}-C_{12}+3 C_{44}\right) / 5$, the corresponding Poisson's ratio $v_{\mathrm{fcc}}=\left(C_{11}+4 C_{12}-2 C_{44}\right) /\left(4 C_{11}+\right.$ $\left.6 C_{12}+2 C_{44}\right)$, as well as $d_{111}=a_{0} / \sqrt{3}, b_{110}=a_{0} / \sqrt{2}$ and $b_{112}=a_{0} / \sqrt{6}$. Consequently, $\boldsymbol{b}_{a}, \gamma_{a}$ and so $\boldsymbol{E}_{a}$ from (4), for $a=1, \ldots, 8$, are determined. In summary, all material parameters in $\psi_{\mathrm{E}}$ from (1), and so in the stress $\boldsymbol{T}$ from (2), are then determined atomistically. 


\begin{tabular}{|c|c|c|c|c|}
\hline & $a_{0}[\AA]$ & $C_{11}[\mathrm{GPa}]$ & $C_{12}[\mathrm{GPa}]$ & $C_{44}[\mathrm{GPa}]$ \\
\hline $\mathrm{Cu}$ & 3.615 & 169.9 & 122.6 & 76.2 \\
\hline $\mathrm{Al}$ & 4.050 & 113.8 & 61.5 & 31.6 \\
\hline
\end{tabular}

Table 1: Zero-temperature (isothermal) lattice constant $a_{0}$ and fcc elastic constants $C_{11}, C_{12}, C_{44}$, for $\mathrm{Cu}$ and $\mathrm{Al}$ calculated from the embedded atom potentials of Mishin et al. (2001) (Cu) and of Mishin et al. (1999) (Al) using the LAMMPS molecular dynamics package (Plimpton, 1995).

\subsection{Stacking fault energy $\psi_{\mathrm{H}}$}

As mentioned above, to model $\psi_{\mathrm{H}}$, Shen and Wang (2004) fit DFT data to the analytical truncated Fourier series representation for this energy from Schoeck $(2001,2003,2005)$. In contrast, a simple numerical scheme utilizing MS data is employed for this purpose here. Given this data, values for $\psi_{\mathrm{H}}$ and its PF derivatives are calculated in PF space and then used in look-up-table fashion during the PF simulation. This results in a speed-up of the calculation of $\psi_{\mathrm{H}}$ by about a factor of 4 in comparison to the Fourier-based approach. In addition, this avoids Fourier-series truncation errors and simplifies energy surface representation up to the desired accuracy.

As usual, calculation of the SFE via MS is based on rigid displacement and relaxation normal to the slip plane. The corresponding energy density $\psi_{\mathrm{H}}\left(\phi_{1}, \phi_{2}\right)$ with respect to the (111) plane (with $\phi_{1}$ and $\phi_{2}$ as in Figure 1) is shown in Figure 2 for $\mathrm{Al}$ (left) and $\mathrm{Cu}$ (middle). Calculation
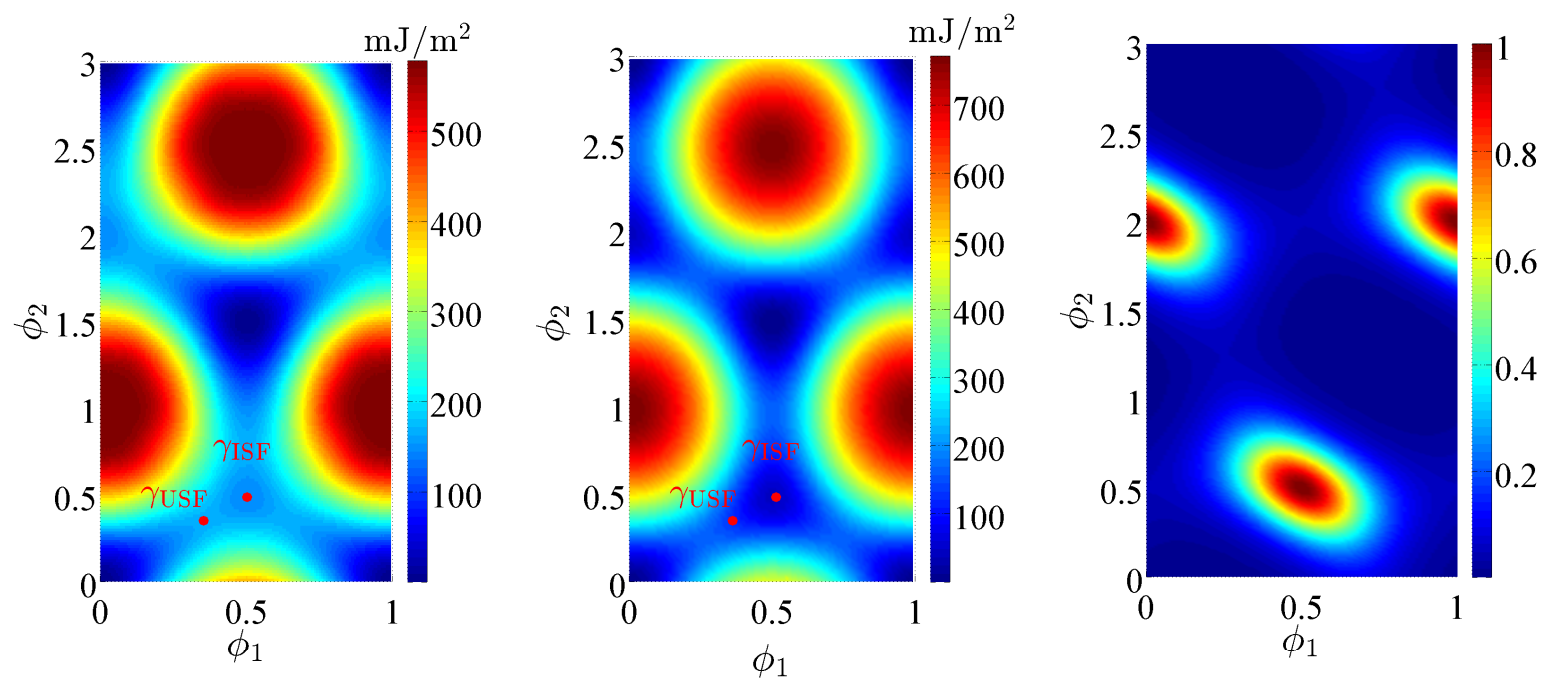

Figure 2: Left, middle: zero-temperature (isothermal) SFE surfaces $\psi_{\mathrm{H}}\left(\phi_{1}, \phi_{2}\right)$ corresponding to Figure 1 for $\mathrm{Al}$ (left) and $\mathrm{Cu}$ (middle) calculated from the corresponding embedded atom potentials of Mishin et al. $(1999,2001)$. The points $\gamma_{\mathrm{USF}}$ and $\gamma_{\mathrm{ISF}}$ represent the unstable and equilibrium energy states, respectively, of instrinsic SFs. Right: periodic function $f_{\mathrm{SF}}\left(\phi_{1}, \phi_{2}\right)$ from (25) used for detection and visualisation of SFs.

of the SFE surfaces shown in Figure 2 employ PF-based parameterization of MS data using 100 points in the $\phi_{1}$ direction, and 200 points in the $\phi_{2}$ direction. Particular values of interest on the 
SFE surface include the equilibrium $\gamma_{\mathrm{ISF}}$ and unstable $\gamma_{\mathrm{USF}}$ values. For $\mathrm{Cu}, \gamma_{\mathrm{ISF}}=45.1 \mathrm{~mJ} / \mathrm{m}^{2}$ and $\gamma_{\mathrm{USF}}=163.4 \mathrm{~mJ} / \mathrm{m}^{2}$. For Al, $\gamma_{\mathrm{ISF}}=145.2 \mathrm{~mJ} / \mathrm{m}^{2}$ and $\gamma_{\mathrm{USF}}=168.2 \mathrm{~mJ} / \mathrm{m}^{2}$. With $\psi_{\mathrm{H}}\left(\phi_{1}, \phi_{2}\right)$ in hand, $\psi_{\mathrm{H}}(\phi)$ is then determined via material symmetry.

In order to visualise SFs in the PF simulation results, it is necessary to define a function capable of detecting partial slip based on values of $\phi_{1}$ and $\phi_{2}$. The periodic function

$$
f_{\mathrm{SF}}\left(\phi_{1}, \phi_{2}\right)=e^{-3 \sin ^{2}\left(\phi_{1}+\phi_{2} / 3-2 / 3\right) \pi} e^{-3 \sin ^{2}\left(2 \phi_{2} / 3-1 / 3\right) \pi}
$$

plotted in Figure 2 is used for this purpose. As shown, the value of $f_{\mathrm{SF}}\left(\phi_{1}, \phi_{2}\right)$ is 1 at the points where an intrinsic SF is located (see Figure 2, left and middle) and decreases toward zero as $\left(\phi_{1}, \phi_{2}\right)$ deviate from SF states. Since this function is periodic, it works for all values of $\left(\phi_{1}, \phi_{2}\right)$ (both negative and positive).

\subsection{Energy scaling}

With $\psi_{\mathrm{E}}$ and $\psi_{\mathrm{H}}$ determined as just discussed, $\kappa_{\mathrm{H} 0}$ and $\kappa_{\mathrm{G} 0}$ follow from (21) and (23), respectively, via (17) and numerical integration (again with $\phi(+\infty)-\phi(-\infty)=1 / \sqrt{2}$ from above). The values of these for $\mathrm{Al}$ and $\mathrm{Cu}$ are listed in Table 2. As discussed above, $g_{0}$ and $l_{0}$ are determined

\begin{tabular}{|c|c|c|c|c|}
\hline & $g_{0} / \mu_{\mathrm{fcc}} d_{111}$ & $l_{0} / d_{111}$ & $\kappa_{\mathrm{H} 0}$ & $\kappa_{\mathrm{G} 0}$ \\
\hline $\mathrm{Cu}$ & 0.055 & 6.289 & 0.429 & 0.479 \\
\hline $\mathrm{Al}$ & 0.064 & 6.053 & 0.303 & 0.468 \\
\hline
\end{tabular}

Table 2: Parameter values for $\mathrm{Cu}$ and $\mathrm{Al}$ determining the energy scaling relations (24). See text for details.

atomistically via MS simulation of the dissociation (13) of 2 straight perfect screw dislocations in a dipole configuration. This results in the formation of 4 partial monopoles (i.e., 2 per perfect monopole). The corresponding simulation is carried out in a box with basis (14) and dimensions $\left(L_{x}, L_{y}, L_{z}\right)=(70 \sqrt{6}, 60 \sqrt{3}, 3 \sqrt{2}) a_{0}=\left(420 b_{112}, 180 d_{111}, 6 b_{110}\right)$. The initial configuration is a perfect straight screw dipole on the (111) plane with line parallel to $\boldsymbol{i}_{z}$ centered at $(y, z)=\left(L_{y} / 2,0\right)$; the monopoles are located initially at $x=L_{x} / 4$ and $x=3 L_{x} / 4$. The displacement field of this dipole is imposed on the atoms using the method for periodic systems described by Bulatov and Cai (2006, Chapter 5). Relaxation to dissociation is carried out via the FIRE algorithm (Bitzek et al., 2006). Since the current implementation of this algorithm in LAMMPS does not include relaxation of the simulation box, this is followed by conjugate-gradient-based relaxation to equilibrium.

The atomic calculation of $g_{0}$ is based on the difference in energy between the equilibrium states of an fcc crystal containing (i) a single dissociated dipole and (ii) no dipole. To this end, consider the rectangular parallelepiped $P$ of length $l_{P}=L_{x}$, width $w_{P}=L_{z}$, and height $h_{P}=2 d_{111}$ formed by the (111) planes at $y=L_{y} / 2 \pm d_{111}$ enclosing the plane containing the cores of the 4 partial dislocations. Then $v_{P}=l_{P} w_{P} h_{P}=2 d_{111} L_{x} L_{z}$ and $a_{P}=w_{P} h_{P}=2 d_{111} L_{z}$. Let $N$ represent the number of atoms in $P$, and $\Delta E_{i}$ the energy difference between the states of atom $i$ in $P$ with and 
without the dissociated dipole. Then

$$
g_{0} \equiv \frac{1}{a_{P}} \sum_{i=1}^{N} \frac{1}{4} \Delta E_{i}
$$

determines the interface energy consistent with (20) for the current case. The factor of 4 in (26) due to the fact that $P$ contains 4 dislocation cores (two partial pairs). Formally at least, $\frac{1}{4} \sum_{i=1}^{N} \Delta E_{i} / v_{P}$ is analogous to $\Delta \psi_{\mathrm{EH}}$ from (15) in the PF context.

The effective core size $l_{0}$ is calculated atomistically via the displacement of the atoms relative to their ideal fcc positions. In the current case of dipole dissociation in the $(x, z)$ plane spanned by $\boldsymbol{i}_{x} \equiv[\overline{1} \overline{1} 2] / \sqrt{6}$ and $\boldsymbol{i}_{z} \equiv[\overline{1} 10] / \sqrt{2}$, the 4 partial dislocation cores are geometrically equivalent, and attention can be restricted to just one. Let $u_{x}$ and $u_{z}$ represent the displacement components of the atoms in the core region of interest relative to the ideal fcc lattice. These are used to calculate the corresponding disregistries $\phi_{x}=u_{x} / b_{x}$ and $\phi_{z}=u_{z} / b_{z}$. In turn, these determine $\phi$ from (17). Finally, this function and its derivative $\partial_{x} \phi(0)$ at $x=0$ are used in (22) to calculate $l_{0}$.

To verify the results in Table 2 , the entire dipole dissociation process is simulated for screw dislocations. The simulation box and initial dislocation configuration are the same as above. Figure 3 displays the resulting disregistry profiles for the screw case. The values of $g_{0}$ and $l_{0}$ obtained from
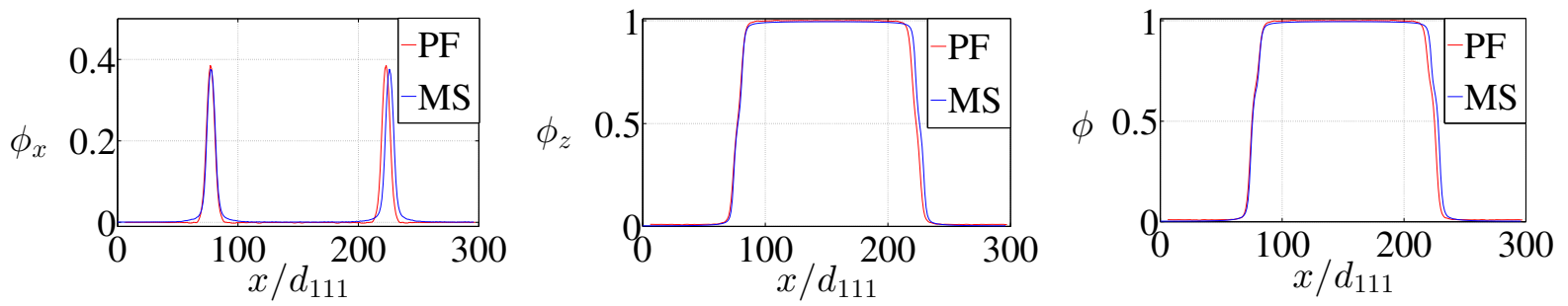

Figure 3: Disregistry profiles for a dissociated screw dipole in $\mathrm{Cu}$ along $\boldsymbol{i}_{x} \equiv[\overline{1} \overline{1} 2] / \sqrt{6}$. Please note that the $x$-axis in these figures refers to the simulation box and not the dislocation line. In particular, $x=0$ on this axis is different that the center of the dislocation $x=0$ in (22) at which $\partial_{x} \phi(x)$ is evaluated.

the PF simulation results are respectively within $1 \%$ and $4 \%$ of the corresponding values from the MS simulation. To validate the identified PF free energy model based on Table 2, edge dipole dissociation has also been simulated. In this case, the simulation box is defined by $\boldsymbol{i}_{x} \equiv[\overline{1} 10] / \sqrt{2}$ and $\boldsymbol{i}_{z} \equiv[\overline{1} \overline{1} 2] / \sqrt{6}$ instead of (14), and dimensions $\left(L_{x}, L_{y}, L_{z}\right)=(120 \sqrt{2}, 60 \sqrt{3}, 3 \sqrt{6} / 2) a_{0}=$ $\left(240 b_{110}, 180 d_{111}, 9 b_{112}\right)$. Corresponding results for $\mathrm{Cu}$ and $\mathrm{Al}$ in the case of edge dissociation are depicted in Figure 4. A further measure of comparison is the equilibrium SF width (Shen and Wang, 2004; Xiang et al., 2008; Hunter et al., 2011). The equilibrium partial dislocation separation and SF width is given by the distance between the two peaks in the gradient of the disregistry (i.e., density) profiles, corresponding to the core positions of the two partials (see Figure 3). Selected results for $\mathrm{Al}$ and $\mathrm{Cu}$ are given in Table 3 . 


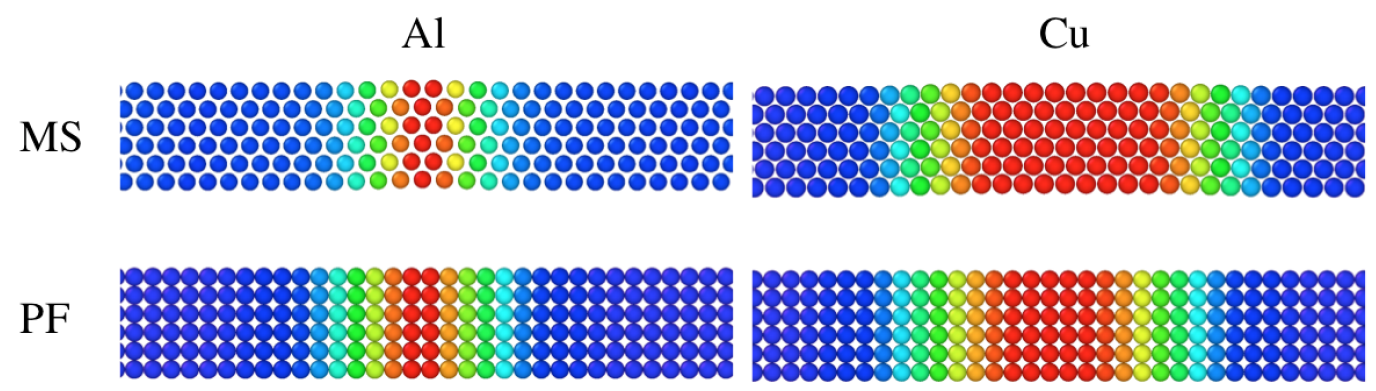

Figure 4: Disregistry fields associated with dissociated straight edge dislocations in Al (left) and $\mathrm{Cu}$ (right). Displayed is the displacement or disregistry component along the line, analogous to the disregistry profile in Figure 3 (middle). The MS results shown are superposed on the atomic lattice, and the PF results on the numerical grid. Ovito (Stukowski, 2010) is used for both visualisations.

\begin{tabular}{|c|c|c|l|}
\hline & edge $[\AA]$ & screw $[\AA]$ & source \\
\hline \multirow{4}{*}{ Al } & 14.0 & 9.3 & PF (this work) \\
& 9.0 & 6.0 & Hunter et al. (2011, 2013) \\
& 12.1 & 7.4 & MS (this work) \\
\hline \multirow{4}{*}{$\mathrm{Cu}$} & 29.2 & 14.6 & PF (this work) \\
& 23.0 & 10.0 & Hunter et al. (2011, 2013) \\
& 37.5 & 13.4 & MS (this work) \\
\hline
\end{tabular}

Table 3: Equilibrium SF widths for $\mathrm{Al}$ and $\mathrm{Cu}$ obtained from the current $\mathrm{PF}$ model and MS results compared with corresponding results from the PN model of Hunter et al. (2011, 2013). Because of its lower (intrinsic) SFE, the SF width in $\mathrm{Cu}$ is larger than that in Al. See text for details.

\section{Application to tensile loading of a dislocation loop}

As a simple application of the atomistically determined PF model developed in the previous sections, consider the simulation of tensile loading of a (initially) perfect dislocation loop on the (111) plane as shown in Figure 5 (left). The simulation box has dimensions of $120 d_{111}$ in all directions. The corresponding results for the final state $\left(\phi_{1}, \phi_{2}\right)$ of the loop after loading are shown in Table 4.

To interpret and understand these results, it is useful to examine the corresponding energy pathways traversed by the loop state in the corresponding (SF) energy landscape shown Figure 5 (right). As well, it is interesting to compare this energetic / thermodynamic picture with the classical purely mechanical force-based one in terms of the corresponding Peach-Köhler force (PKF). To do this in a meaningful fashion, it is necessary to establish the relation between the models for the Burgers vector tacitly assumed in the PF model via the residual distortion relation (3)-(4) and that in the PKF. This is done in Appendix B and summarized in Figure B.1. Given this, the glide component of the PKF from (B.4) is calculated on each partial dislocation loop for the three loading cases in Table 4. The results are shown in Figure 6. Consider first the case 

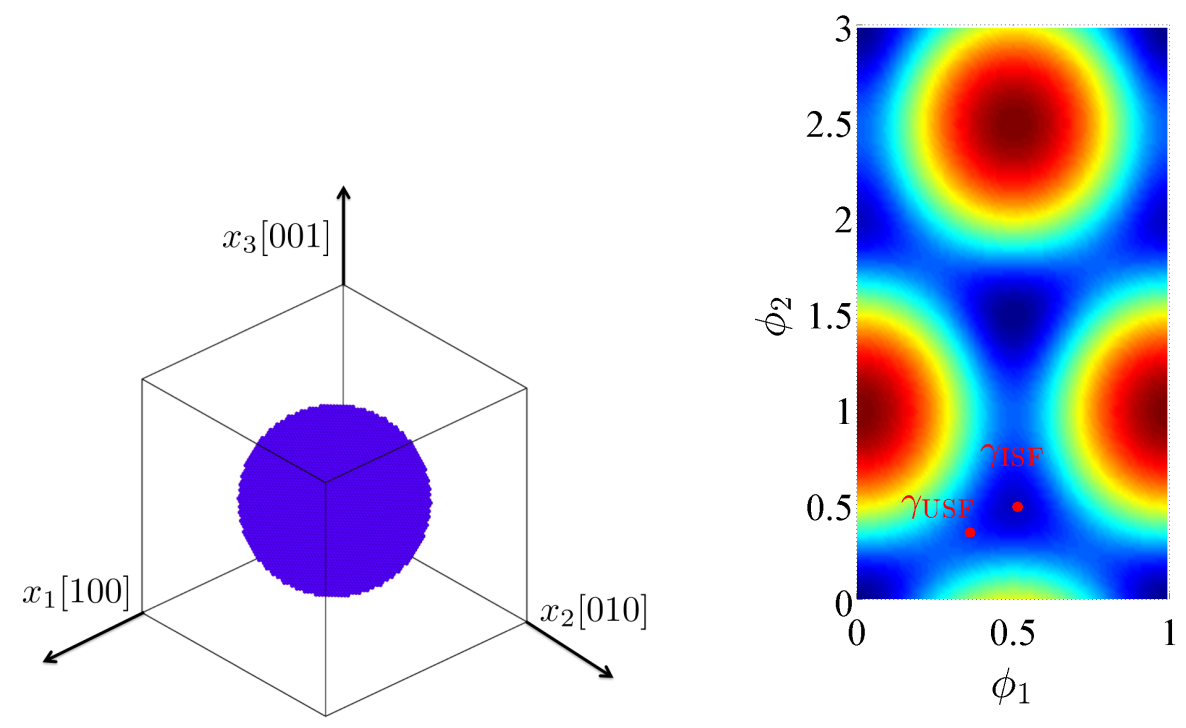

Figure 5: Left: initially perfect dislocation loop $\left(\phi_{1}, \phi_{2}\right)=(1,0)$ of radius $r=30 \sqrt{2} d_{111}$ on (111). Right: SFE surface $\psi_{\mathrm{H}}\left(\phi_{1}, \phi_{2}\right)$ of $\mathrm{Cu}$ from Figure 2 (middle). The initial loop state $\left(\phi_{1}, \phi_{2}\right)=(1,0)$ corresponds to $\mathbf{A B}$ in Figure 1 and in (13). See text for details.
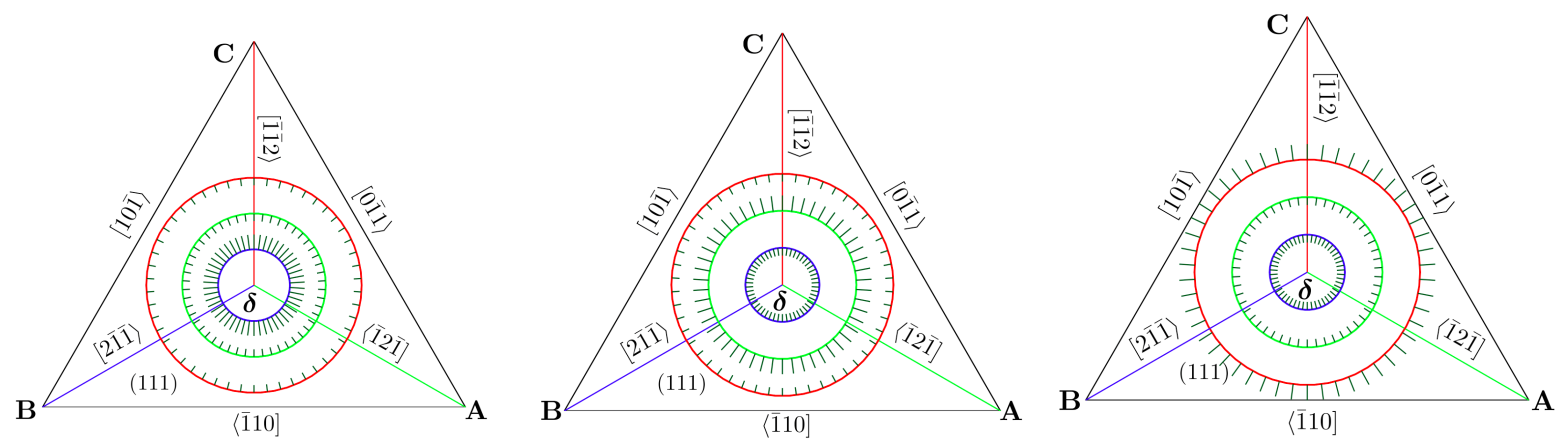

Figure 6: PKF on the three (111) partial dislocations depending on loading. Left: $T_{11}$ loading. Middle: $T_{22}$ loading. Right: $T_{33}$ loading. Red: [ $[\overline{1} \overline{1} 2\rangle$ partial. Green: [ $[\overline{1} 2 \overline{1}\rangle$ partial. Blue: $[2 \overline{1} \overline{1}\rangle$ partial. Outward-directed (inward-directed) tick marks indicate an expansive (contractive) PKF; tick length indicates (relative) magnitude.

of $T_{11}$ loading in Table 4. As implied in Figure 6 (left), this results in contractive PKFs on the partials in the dissociation (13). Note that the PKF associated with $[2 \overline{1} \overline{1}\rangle$ (i.e., $\mathbf{B} \delta$ : blue in Figure 6 , left) is expansive; since the negative of this (i.e., $\delta \mathbf{B}$ ) appears in the dissociation (13), however, the force changes sign. Consequently, such loading enhances the (contractive) loop self-stress at any loading level and results in its annihilation in both $\mathrm{Cu}$ and $\mathrm{Al}$. The corresponding energy pathway from $\left(\phi_{1}, \phi_{2}\right)=(1,0)$ to $\left(\phi_{1}, \phi_{2}\right)=(0,0)$ in Figure 5 (right) traverses the intermediate state $\left(\phi_{1}, \phi_{2}\right)=\left(\frac{1}{2}, \frac{1}{2}\right)$, as implied by (13). In contrast to this, $T_{22}$ loading results in a PKF on both partials which is expansive, opposing as such the (contractive) self-stress. As shown in Table 


\begin{tabular}{|c|c|c|c|c|c|c|c|}
\hline & \multicolumn{6}{|c|}{ Stress magnitude (in units of $\mu_{\text {fcc }}$ ) } \\
\hline & & 0.02 & 0.05 & 0.10 & 0.15 & 0.20 & 0.25 \\
\hline \multirow{3}{*}{$\mathrm{Al}$} & $T_{11}$ & $(0,0)$ & $(0,0)$ & $(0,0)$ & $(0,0)$ & $(0,0)$ & $(0,0)$ \\
\hline & $T_{22}$ & $(0,0)$ & $(1,0)$ & $(1,0)$ & $(1,0)$ & $(1,0)$ & $(1,0)$ \\
\hline & $T_{33}$ & $(0,0)$ & $(0,0)$ & $(0,0)$ & $(0.5,1.5)$ & $(0.5,1.5)$ & $(0.5,1.5)$ \\
\hline \multirow{3}{*}{$\mathrm{Cu}$} & $T_{11}$ & $(0,0)$ & $(0,0)$ & $(0,0)$ & $(0,0)$ & $(0,0)$ & $(0,0)$ \\
\hline & $T_{22}$ & $(0,0)$ & $(1,0)$ & $(1,0)$ & $(1,0)$ & $(1,0)$ & $(1,0)$ \\
\hline & $T_{33}$ & $(0,0)$ & $(0.5,0.5)$ & $(0.5,0.5)$ & $(0.5,1.5)$ & $(0.5,1.5)$ & $(0.5,1.5)$ \\
\hline
\end{tabular}

Table 4: Final state $\left(\phi_{1}, \phi_{2}\right)$ of the initially perfect loop (Figure 5, left) in $\mathrm{Al}$ and $\mathrm{Cu}$ subject to tensile loading. Here, $T_{11}, T_{22}$, and $T_{33}$ are the stress components in the [100], [010], and [001], directions, respectively (see Figure 5, left).

4, below a value for $T_{22}$ of $0.05 \mu_{\mathrm{fcc}}$, this opposing force is insufficient to prevent loop collapse. Above this level, however, $T_{22}$ results in further expansion. In addition, the fact that the PKF is of different magnitude on the leading and trailing partials (see length of tick marks in Figure 6, center) leads to a deviation of the SF width from its equilibrium value. As already seen in Table 3, because of its lower (intrinsic) SFE, the SF width in $\mathrm{Cu}$ is larger than that in $\mathrm{Al}$.

The most interesting of the three loading cases in Table 4 is the final case of $T_{33}$ loading. As shown in Figure 6 (right), this results in an expansive PKF on $\delta \mathbf{B}$ (blue) and a contractive PKF on $\mathbf{A} \boldsymbol{\delta}$ (green). As before, an expansive PKF works against the self-stress of the loop, and a contractive one enhances it. Consequently, if the level of loading is too low, the expansive PKF is insufficient to prevent loop annihilation. As the PKF on $\mathbf{A} \boldsymbol{\delta}$ and $\boldsymbol{\delta} \mathbf{B}$ is equal and opposite, the net PKF is approximately zero. In terms of thermodynamic state $\left(\phi_{1}, \phi_{2}\right)$, the results in Table 4 show that the initial state $\left(\phi_{1}, \phi_{2}\right)=(1,0)$ transforms to $\left(\phi_{1}, \phi_{2}\right)=(0,0)$ in both $\mathrm{Al}$ and $\mathrm{Cu}$. The loop in $\mathrm{Al}$ remains in this state up to $T_{33}=0.15 \mu_{\text {fcc }}$, at which point it transforms to $\left(\phi_{1}, \phi_{2}\right)=(0.5,1.5)$. On the other hand, the loop in $\mathrm{Cu}$ remains in this state up to $T_{33}=0.05 \mu_{\mathrm{fcc}}$, where it transforms to $\left(\phi_{1}, \phi_{2}\right)=(0.5,0.5)$. Note that this latter state represents an extended SF. Because Al has a relatively high SFE, this state is energetically unfavorable for the loop in Al. As shown by the results in Table 4, the next energetically favorable state for this loop is the perfect state $\left(\phi_{1}, \phi_{2}\right)=$ $(0.5,1.5)$ at $T_{33}=0.15 \mu_{\mathrm{fcc}}$. As depicted in Figure 5 (right), from the geometric point of view, this is CB. This can also be understood in terms of the respective PKFs on the partials involved by looking at the possible reaction

$$
\mathrm{A} \delta+\delta \mathbf{B} \longrightarrow \mathbf{A} \delta+\delta \mathbf{C}+\mathbf{C} \delta+\delta \mathbf{B} .
$$

Indeed, since the PKF on $\mathbf{A} \boldsymbol{\delta}$ and $\boldsymbol{\delta} \mathbf{C}$ is contractive, the sum of these is as well, and $\mathbf{A C}$ vanishes. On the other hand, $\mathbf{C} \boldsymbol{\delta}$ and $\boldsymbol{\delta} \mathbf{B}$ are expansive, resulting in expansion of $\mathbf{C B}$. For the case of $\mathbf{C u}$, as shown by Table 4 , the extended SF state $\left(\phi_{1}, \phi_{2}\right)=(0.5,0.5)$ at $T_{33}=0.05 \mu_{\text {fcc }}$ also transforms to the perfect state $\left(\phi_{1}, \phi_{2}\right)=(0.5,1.5)$ at $T_{33}=0.15 \mu_{\mathrm{fcc}}$. As in the case of Al, this represents the reaction (27). Because the SFE of $\mathrm{Cu}$ is lower, this does not result in the formation of perfect dislocations; rather, the partial-SF combinations $\mathbf{A} \boldsymbol{\delta}+\boldsymbol{\delta} \mathbf{C}$ and $\mathbf{C} \boldsymbol{\delta}+\boldsymbol{\delta} \mathbf{B}$ are favored. Since the PKF 
configuration is the same as in the case of $\mathrm{Al}, \mathbf{A} \boldsymbol{\delta}+\boldsymbol{\delta} \mathbf{C}$ vanishes, and $\mathbf{C} \boldsymbol{\delta}+\boldsymbol{\delta} \mathbf{B}$ expands.

The evolution of loop state in both $\mathrm{Al}$ and $\mathrm{Cu}$ just discussed is displayed in Figure 7. The

$\mathrm{Al}$
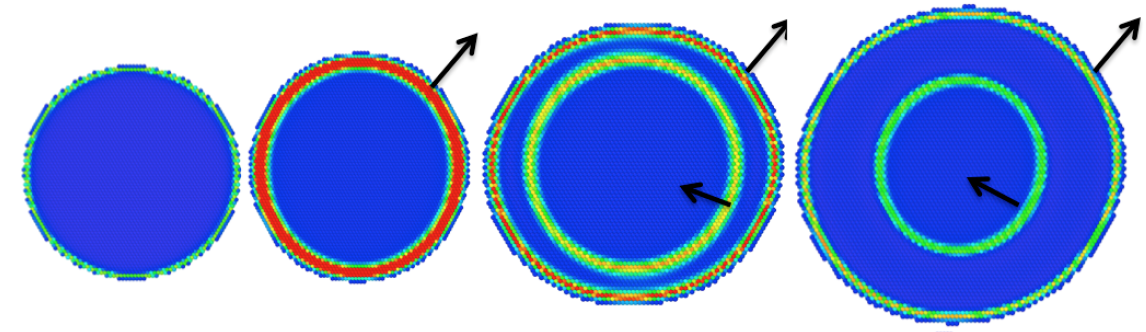

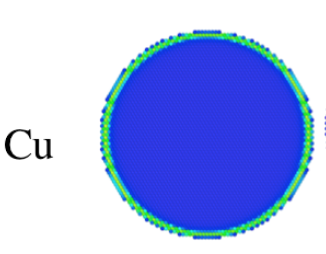

1

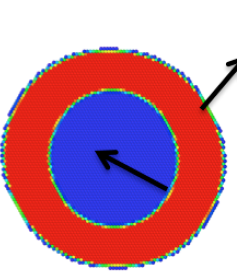

2

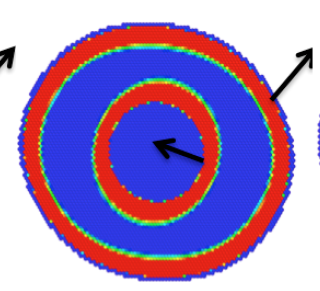

3

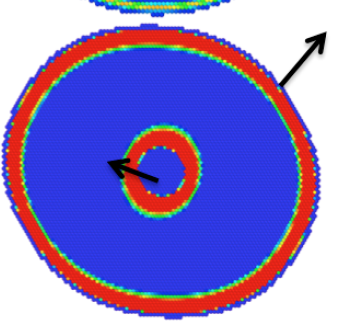

4

Figure 7: Stages of dynamic dislocation loop transformation in $\mathrm{Al}$ and $\mathrm{Cu}$ on (111) during $T_{33}$ loading. Depicted are 4 intermediate stages during the transformation of the initial state $\left(\phi_{1}, \phi_{2}\right)=$ $(1,0)$ (Stage 1) to $\left(\phi_{1}, \phi_{2}\right)=(0.5,1.5)\left(\right.$ Stage 4) at $T_{33}=0.15 \mu_{\text {fcc }}$ as in Table 4. The black arrows indicate the direction of motion of the line. Blue: perfectly slipped crystal. Red: SF. Green: dislocation line. See text for details.

transition from Stage 1 (i.e., the intial state $\left.\left(\phi_{1}, \phi_{2}\right)=(1,0)\right)$ to Stage 2 is the dissociation (13); that from Stage 2 to Stage 3 is the reaction (27). In going from Stage 3 to 4, further shrinkage of the inner partial loops, and expansion of the outer partial loops (as shown by the black arrows), takes place in both $\mathrm{Al}$ and $\mathrm{Cu}$. The results for this last $T_{33}$ case are also (at least academically) interesting in that they show how external loading can transform a perfect dislocation $\mathbf{A B}$ through the series of core reactions (13) and (27) into another perfect dislocation $\mathbf{C B}$, resulting in perfect slip. In contrast to dissociation into partials, such "dissociation" (transformation) into perfect dislocations is energetically unfavourable unless the system is externally loaded and no other more favorable mechanism is available. In real systems, of course, such a more favorable mechanism generally exists.

\section{Summary \& discussion}

In the current work, a dynamic phase field (PF) model for dislocation dissociation, stacking fault (SF) formation, slip and reactions in fcc single crystals has been developed and identified with the help of atomistic methods. To this end, periodic microelasticity (e.g., Khachaturyan, 1983; Wang et al., 2001; Bulatov and Cai, 2006, Chapter 11) has been combined with a PF-based model for residual strain resulting from dislocation lines (cores) and SFs. The energy model is formulated with the help of the interface energy approach of Cahn and Hilliard (1958), representing 
a type of local or generalized gradient approximation in the context of classical density functional theory (e.g., Evans, 1979; Davis, 1996). For the current dislocation case, the homogeneous part of this energy is related to homogeneous atomic displacement across the slip plane during dislocation slip and so to the (generalized) SFE (Vitek, 1968); the "gradient" part is due to deviations from homogeneity such as occurs in the dislocation core (something also predicted by DFT-based core modeling: Iyer et al., 2015). As shown in this work, interface energy modeling facilitates global energy scaling, which in turn can be exploited to identify the PF model completely using atomistic data. In particular, the resulting gradient energy is material specific and related to the physical core. To demonstrate the application of the current approach, PF models were identified for fcc Al and $\mathrm{Cu}$ with the help of atomistic data and applied to the case of dynamic tensile loading of a dislocation loop in these materials. In the process, the thermodynamic PF picture in terms of energetic favorability and thermodynamic driving forces was compared to, and shown to be consistent with, the more standard mechanical picture in terms of the Peach-Köhler force. The example cases of $\mathrm{Al}$ and $\mathrm{Cu}$ demonstrate the ease of calculating atomistic input data for the current PF model and its predicative capability in the simulation of dislocation processes which are difficult to investigate via MD. The use of an atomic potential and MS in this work to determine this input data was for simplicity only; indeed, if available, more exact DFT-based input data can (and should) be used.

As shown in the current work, with the help of basic processes like dissociation, the interface energy model and scaling approach facilitate the atomistic determination of the (continuum) PF free energy as a mininum-difference approximation of the (discrete) atomic energy. Indeed, the PF free energy determined in this fashion represents an optimal continuum approximation of the exact atomistic (physical) energy. In this sense, the resulting atomistically determined PF model represents a type of model-order reduced approximation of more accurate atomistic modeling. As in other analogous cases, such reduction facilitates in particular the modeling of larger systems and longer timescales. Besides this, the pursuit of a PF approach as a continuum approximation or model reduction of more accurate atomistic modeling facilitates as well model generalization in the spirit of computation material design. As is well-known, in this framework, one can account for the effect of chemistry and temperature on the energy landscape, and so on dislocation and related deformation processes whose driving forces are determined by this landscape.

A number of further model developments are currently under investigation. One possibility in this regard is the application of the more general scaling method of Reina et al. (2014) in the current context. Rather than on thermodynamic equilibrium, their method is based on steady state, applies to both energetics and kinetics (i.e., mobility), and can be used to identify PF models using atomistic data from molecular dynamics (MD) simulations. As has been alluded to above, another further development involves the use of ab initio DFT-based methods (e.g., Woodward et al., 2008), instead of an interatomic potential and MS or MD. Going even further, the generalization of such methods to larger systems with the help of coarse-graining methods (e.g., quasi-continuum: Iyer et al., 2015) may offer the possibility of true "concurrent" PF-DFT modeling of dislocation processes in the future.

Finally, it should be noted that the determination of the PF model in atomistic or ab initio fashion as developed in this work is almost certainly more subtle than the current treatment would imply. This is related for example to the fact that there exist qualitative differences between atomic modeling of dislocation processes and the current PF modeling. For example, atomic displace- 
ments modeled via DFT or an atomic potential and molecular statics are intrinsically non-linear, both geometrically and physically (especially in the core region). Another difference lies in the form of the respective energy models. Indeed, whereas in the atomistic or DFT case the energy is modeled via a single unified form (e.g., an atomic potential), in the PF model the energy is split into parts (i.e., elastic, SF contribution, etc.). That this may not in fact be necessary in a PF-based model is shown for example by the phase-field crystal approach (e.g., Wu et al., 2010). In any case, these and other issues represent work in progress to be reported on in the future.

Acknowledgements. We thank the reviewers for their very constructive comments and criticism which resulted in major improvement of the presentation. Financial support by the German Science Foundation (DFG) of Subproject A10 in the Collaborative Research Center SFB 761 is gratefully acknowledged.

\section{References}

Allen, S. M., Cahn, J. W., 1979. A macroscopic theory for antiphase boundary motion and its application to antiphase domain coarsening. Acta Metallurgica 27, 1085-1095.

Balluffi, R. W., 2012. Introduction to Elasticity for Crystal Defects. Cambridge University Press.

Bitzek, E., Gahler, F., Koskinen, P., Moseler, M., Gumbsch, P., 2006. Structural relaxation made simple. Physical Review Letters 97, 170201.

Brown, C. M., Dreyer, W., Müller, W., 2002. Discrete fourier transforms and their application to stress-strain problems in composite mechanics: a convergence study. Proceedings of the Royal Society of London A 458 (1967-1987).

Bulatov, V. V., Cai, W., 2006. Computer Simulation of Dislocations. Oxford Series on Materials Modelling. Oxford.

Cahn, J. W., Hilliard, J. E., 1958. Free energy of a non-uniform system. i. interfacial energy. Journal of Chemical Physics 28, 258-267.

Canuto, C., Hussaini, M. Y., Quateroni, A., Zang, T. A., 1988. Spectral Methods in Fluid Dynamics. Springer Series in Computational Physics. Springer.

Chen, L. Q., Shen, J., 1998. Applications of semi-implicit fourier-spectral method to phase field equations. Computer Physics Communications 108, 147-158.

Christian, J. W., Mahajan, S., 1995. Deformation twinning. Progress in Materials Science 39, 1-157.

Davis, H. T., 1996. Statistical Mechanics of Phases, Interfaces, and Thin Films. Advances in Interfacial Engineering Series. Wiley-VCH, New York, Ch. 9.1: Calculus of variations and functional derivatives, pp. 425-435.

Dreyer, W., Müller, W., 2000. A study of the coarsening of tin/lead solders. International Journal of Solids and Structures 37, 3841-3871.

Eisenlohr, P., Diehl, M., Lebensohn, R. A., Roters, F., 2013. A spectral method solution to crystal elasto viscoplasticity at finite strains. International Journal of Plasticity 46, 37-53.

Eshelby, J., 1961. Elastic inclusions and inhomogeneities. Progress in Solid Mechanics 2, 89-140.

Eshelby, J. D., 1970. Energy relations and the energy-momentum tensor in continuum mechanics. In: Kanninen, M., Alder, W., Rosenfeld, A., Jaffe, R. (Eds.), Inelastic behavior in solids. Academic Press, pp. 77-115.

Evans, R., 1979. The nature of the liquid-vapour interface and other topics in the statistical mechanics of non-uniform, classical fluids. Advances in Physics 28, 143-200.

Gottlieb, D., Orszag, S. A., 1977. Numerical Analysis of Spectral Methods: Theory and Application. SIAM.

Hirth, J. P., Lothe, J., 1982. Theory of Dislocations, 2nd Edition. Wiley.

Hunter, A., Beyerlein, I. J., Germann, T. C., Koslowski, M., 2011. Influence of the stacking fault energy surface on partial dislocations in fcc metals with a three dimensional phase field dynamics model. Physical Review B 84, 144108.

Hunter, A., Zhang, R. F., Beyerlein, I. J., Germann, T. C., Koslowski, M., 2013. Dependence of equilibrium stacking fault width in fcc metals on the gamma-surface. Modelling and Simulation in Materials Science and Engineering 21,025015 (19pp). 
Iyer, M., Radhakrishnan, B., Gavini, V., 2015. Electronic-structure study of an edge dislocation in aluminum and the role of macroscopic deformations on its energetics. Journal of the Mechanics and Physics of Solids, in press.

Khachaturyan, A. G., 1983. Theory of Structural Transformations in Solids. John Wiley.

Khachaturyan, A. G., Semenovskaya, S., Tsakalakos, T., 1995. Elastic strain energy of inhomogeneous solids. Physical Review B 52 (22), 15909-15919.

Kröner, E., 1959. Allgemeine Theorie der Versetzungen und Eigenspannungen. Archive for Rational Mechanics and Analysis 4 ((Supplement 1)), 273-334.

Landau, L. D., Lifshitz, E. M., 1970. Theory of Elasticity, 2nd Edition. Pergamon.

Li, S., Wang, G., 2008. Introduction to Micromechanics and Nanomechanics. World Scientific.

Mishin, Y., Farkas, D., Mehl, M. J., Papaconstantopoulos, D. A., 1999. Interatomic potentials for monoatomic metals from experimental data and ab initio calculations. Physical Review B 59, 3393.

Mishin, Y., Mehl, M. J., Papaconstantopoulos, D. A., Voter, A., Kress, J., 2001. Structural stability and lattice defects in copper: ab initio, tight-binding, and embedded-atom calculations. Physical Review B 63, 1-16.

Moulinec, H., Suquet, P., 1994. A fast numerical method for computing the linear and nonlinear mechanical properties of composites. Comptes Rendus de l'Académie des Sciences Paris 318, 1417-1423.

Mura, T., 1987. Micromechanics of Defects in Solids. Martinus Nijhoff.

Nemat-Nasser, S., Hori, M., 1999. Micromechanics: Overall Properties of Heterogeneous Materials. Elsevier.

Plimpton, S., 1995. Fast parallel algorithms for short-range molecular dynamics. Journal of Computational Physics 117, 1-42.

Reina, C., Sandoval, L., Marian, J., 2014. Mesoscale computational study of the nanocrystallization of amorphous Ge via a self-consistent atomistic phase-field model. Acta Materialia 77, 335-351.

Schoeck, G., 2001. The core structure, recombination energy and Peierls energy for dislocations in Al. Philosophical Magazine A 81, 1161.

Schoeck, G., 2003. The emission of dislocations from crack tips - a critical assessment. Material Science and Engineering A 356, 93-101.

Schoeck, G., 2005. The peierls model: progress and limitations. Material Science and Engineering A 400-401, 7-17.

Schoeck, G., 2006. The core structure of dislocations: peierls model versus atomic simulations. Acta Materialia 54, 4865-4870.

Shen, C., Ju, L., Wang, Y., 2014. Predicting structure and energy of dislocations and grain boundaries. Acta Materialia $74,125-131$.

Shen, C., Wang, Y., 2003. Phase field modeling of dislocation networks. Acta Materialia 51, 2595-2610.

Shen, C., Wang, Y., 2004. Incorporation of gamma-surface to phase field model of dislocations: simulation dislocation dissociation in fcc crystals. Acta Materialia 52, 683-691.

Stukowski, A., 2010. Visualization and analysis of atomistic simulation data with ovito - the open visualization tool. Modelling and Simulation in Materials Science and Engineering 18, 015012.

Suquet, P., 1997. Continuum Micromechanics. Vol. 377 of CISM International Center for Mechanical Sciences. Springer.

Swygenhoven, H. V., Derlet, P. M., Frøseth, A. G., 2006. Nucleation and propagation of dislocation in nanocrystalline fcc metals. Acta Materialia 54, 1975-1983.

Teodosiu, C., 1982. Elastic Theory of Crystals. North Holland.

Vitek, V., 1968. Intrinsic stacking faults in body-centered cubic crystals. Philosophical Magazine 18, 773-786.

Šilhavý, M., 1997. The Mechanics and Thermodynamics of Continuous Media. Springer.

Wang, Y., Li, J., 2010. Phase field modeling of defects and deformation. Acta Materialia 58, 1212-1235.

Wang, Y. U., Jin, Y. M., Cutiño, A. M., Khachaturyan, A. G., 2001. Nanoscale phase field microelasticity theory of dislocations: model and 3d simulations. Acta Materialia 49, 1847-1857.

Woodward, C., Trinkle, D. R., Hector, L. G., Olmsted, D. L., 2008. Prediction of dislocation cores in aluminum from density functional theory. Physical Review Letters 100, 045507.

Wu, K.-A., Adland, A., Karma, A., 2010. Phase-field-crystal model for fcc ordering. Physical Review E 81, 061601.

Xiang, Y., Wei, H., Ming, P., E, W., 2008. A generalized Peirls-Nabarro model for curved dislocations and core structures of dislocation loops in Al and Cu. Acta Materialia 56, 1447-1460.

Zimmerman, J. A., Gao, H., Abraham, F. F., 2000. Generalized stacking fault energies for embedded atom fcc metals. 
Modelling and Simulation in Materials Science and Engineering 8, 103-115.

\section{A. Selected algorithmic \& numerical details}

Given the restriction to periodic fields and boundary conditions in this work, the algorithmic formulation and numerical solution of the corresponding coupled initial-boundary-value problem (IBVP) may be based on discrete Fourier, and more generally spectral, methods (e.g., Gottlieb and Orszag, 1977; Canuto et al., 1988). These have a long tradition in periodic microelasticity (e.g., Khachaturyan, 1983; Mura, 1987; Khachaturyan et al., 1995; Wang et al., 2001; Li and Wang, 2008) and geometrically linear solid mechanics (e.g., Moulinec and Suquet, 1994; Suquet, 1997; Nemat-Nasser and Hori, 1999). More recently, they have been extended to the geometrically nonlinear realm (e.g., Eisenlohr et al., 2013) as well. For completeness, a brief summary of the spectral algorithmic formulation of the current model is provided here. In a nutshell, this is based on fixedpoint iteration (e.g., Suquet, 1997; Dreyer and Müller, 2000; Brown et al., 2002) for solution of (5) in Fourier space combined with semi-implicit time-integration of (6) as based on first-order Adams-Bashforth treatment of $\partial_{\phi_{a}} \psi$ (e.g., Chen and Shen, 1998).

Consider first the algorithmic form of quasi-static momentum balance (5). The form of this applicable to strain-based fixed-point iteration is obtained from the convolution

$$
\boldsymbol{G}_{\mathrm{H}} * \operatorname{div} \boldsymbol{T}=\mathbf{0}
$$

of (5) with the Green function $\boldsymbol{G}_{\mathrm{H}}$ for a homogeneous reference medium with elastic stiffness $C_{\mathrm{H}}$. Fourier transform of (A.1) yields $\hat{\boldsymbol{G}}_{\mathrm{H}} \hat{\boldsymbol{T}}_{\boldsymbol{l}} \boldsymbol{k}=\mathbf{0}$ for all non-zero wavenumbers $\boldsymbol{k} \neq \mathbf{0}$ with

$$
\boldsymbol{a} \cdot \hat{\boldsymbol{G}}_{\mathrm{H}}^{-1}(\boldsymbol{k}) \boldsymbol{b}:=\boldsymbol{a} \otimes \boldsymbol{k} \cdot C_{\mathrm{H}}[\boldsymbol{b} \otimes \boldsymbol{k}]
$$

as usual for $\boldsymbol{k} \neq \mathbf{0}$ and all $\boldsymbol{a}, \boldsymbol{b}$. Then $\hat{\boldsymbol{G}}_{\mathrm{H}} \hat{\boldsymbol{T}}_{l} \boldsymbol{k} \otimes \imath \boldsymbol{k}=-\hat{\boldsymbol{G}}_{\mathrm{H}} \hat{\boldsymbol{T}} \boldsymbol{k} \otimes \boldsymbol{k}=\mathbf{0}$ represents an implicit relation for $\boldsymbol{H}=\nabla \boldsymbol{u}$, and

$$
-\hat{\boldsymbol{\Gamma}}_{\mathrm{H}} \hat{\boldsymbol{T}}:=-\operatorname{sym} \hat{\boldsymbol{G}}_{\mathrm{H}} \hat{\boldsymbol{T}} \boldsymbol{k} \otimes \boldsymbol{k}=\mathbf{0}
$$

one for $\boldsymbol{E}=\operatorname{sym} \nabla \boldsymbol{u}$, again for $\boldsymbol{k} \neq \mathbf{0}$. Here, $\boldsymbol{\Gamma}_{\mathrm{H}}$ is the Lippmann-Schwinger operator (e.g., Suquet, 1997) induced by $\boldsymbol{G}_{\mathrm{H}}$. In the fixed-point context, then, $\hat{\boldsymbol{E}}^{(i+1)}=\hat{\boldsymbol{E}}^{(i)}-\hat{\boldsymbol{\Gamma}}_{\mathrm{H}} \hat{\boldsymbol{T}}^{(i)}$ follows from (A.3) for the $i^{\text {th }}$ iterative update step. Since $C_{\mathrm{E}}$ is homogeneous and constant in the current single crystal context, $C_{\mathrm{H}}$ can in fact be identified with $C_{\mathrm{E}}$ here, resulting in a further simplification of (A.3). Indeed, with $\hat{\boldsymbol{\Gamma}}_{\mathrm{H}} \hat{\boldsymbol{T}}=\hat{\boldsymbol{\Gamma}}_{\mathrm{E}} \boldsymbol{C}_{\mathrm{E}} \hat{\boldsymbol{E}}-\hat{\boldsymbol{\Gamma}}_{\mathrm{E}} \boldsymbol{C}_{\mathrm{E}} \hat{\boldsymbol{E}}_{\mathrm{R}}$ via the Fourier transform of (2), and $C_{\mathrm{E}}[\hat{\boldsymbol{u}} \otimes \imath \boldsymbol{k}] \boldsymbol{k}=\hat{\boldsymbol{G}}_{\mathrm{E}}^{-1} \hat{\boldsymbol{u}}$ from (A.2), we have $\hat{\boldsymbol{\Gamma}}_{\mathrm{E}} C_{\mathrm{E}} \hat{\boldsymbol{E}}=\operatorname{sym} \hat{\boldsymbol{G}}_{\mathrm{E}} C_{\mathrm{E}}[\hat{\boldsymbol{u}} \otimes \imath \boldsymbol{k}] \boldsymbol{k} \otimes \boldsymbol{k}=\operatorname{sym} \hat{\boldsymbol{u}} \otimes \imath \boldsymbol{k}=\hat{\boldsymbol{E}}$. Consequently, (A.3) reduces to

$$
\hat{\boldsymbol{E}}(\boldsymbol{k})= \begin{cases}\hat{\boldsymbol{\Gamma}}_{\mathrm{E}}(\boldsymbol{k}) C_{\mathrm{E}} \hat{\boldsymbol{E}}_{\mathrm{R}}(\boldsymbol{k}) & \boldsymbol{k} \neq \mathbf{0} \\ \overline{\boldsymbol{E}} & \boldsymbol{k}=\mathbf{0}\end{cases}
$$

for the equilibrium strain field via (3) and (4), i.e., when $C_{\mathrm{E}}$ is constant. The corresponding stress field

$$
\hat{\boldsymbol{T}}(\boldsymbol{k})= \begin{cases}C_{\mathrm{E}}\left[\hat{\boldsymbol{E}}(\boldsymbol{k})-\hat{\boldsymbol{E}}_{\mathrm{R}}(\boldsymbol{k})\right]=\left(C_{\mathrm{E}} \hat{\boldsymbol{\Gamma}}_{\mathrm{E}}(\boldsymbol{k}) C_{\mathrm{E}}-C_{\mathrm{E}}\right) \hat{\boldsymbol{E}}_{\mathrm{R}}(\boldsymbol{k}) & \boldsymbol{k} \neq \mathbf{0} \\ C_{\mathrm{E}}\left[\overline{\boldsymbol{E}}-\hat{\boldsymbol{E}}_{\mathrm{R}}(\mathbf{0})\right] & \boldsymbol{k}=\mathbf{0}\end{cases}
$$


is equivalent to the relation obtained for example by Bulatov and Cai (2006, Equation (11.55)). Note that $\hat{\boldsymbol{E}}_{\mathrm{R}}(\mathbf{0})$ is determined by $\overline{\boldsymbol{\phi}}=\hat{\boldsymbol{\phi}}(\mathbf{0})$ representing the volume average of this over the unit cell.

Consider next the algorithm for numerical solution of the Ginzburg-Landau relation (6). As mentioned above, this is based on semi-implicit time integration (e.g., Chen and Shen, 1998). More specifically, first-order backward-difference approximation of $\dot{\phi}_{a}$, and first-order AdamsBashforth approximation of $\partial_{\phi_{a}} \psi$, is employed. Let $I=\bigcup_{n=0}\left[t_{n}, t_{n+1}\right]$ represent the discretization of the time interval $I \subset \mathbb{R}$ of interest. For simplicity, assume that each subinterval $\left[t_{n}, t_{n+1}\right]$ of $I$ is of the same duration $\Delta t=t_{n+1}-t_{n}$. Consider an arbitrary subinterval $\left[t_{n}, t_{n+1}\right]$. Assume as usual that $\boldsymbol{E}_{n}, \boldsymbol{\phi}_{n}\left(\boldsymbol{\phi}:=\left(\phi_{1}, \ldots\right)\right)$, and so $\left(\partial_{\phi} \psi\right)_{n}:=\left(\partial_{\phi_{1}} \psi, \ldots\right)_{n}$, are known from the the solution obtained at the end of the previous time step. Fourier transformation of (6) and rearrangement then results in the linear system

$$
\hat{\mathbf{M}} \hat{\boldsymbol{\phi}}_{n+1}=\hat{\boldsymbol{\phi}}_{n}-m_{0} \Delta t \widehat{\left(\partial_{\boldsymbol{\phi}} \psi\right)_{n}}
$$

to solve for $\hat{\boldsymbol{\phi}}_{n+1}$ with $\hat{M}_{a b}:=\delta_{a b}+m_{0} \Delta t \psi_{\mathrm{G} 0} \boldsymbol{k} \cdot \boldsymbol{N}_{a b}^{g} \boldsymbol{k}$ and $\boldsymbol{N}_{a b}^{g}$ given by (12). Note that (A.6) is a direct update; no iteration is required. In addition, note that $\hat{\mathbf{M}}$ depends only on (constant) material data and the chosen discretization in wavenumber space. For a given material and discretization, then, it need be calculated only once at the start as part of the initial data.

As discussed in the text, all material properties $\psi_{\mathrm{H} 0}, \psi_{\mathrm{G} 0}, m_{0}$, as well as $\boldsymbol{H}_{a}$ and $\boldsymbol{N}_{a b}^{\phi}$ for $a, b=$ $1, \ldots, p$, are known and constant. In addition, this holds for $C_{\mathrm{E}}$ and so for $C_{\mathrm{H}}=C_{\mathrm{E}}$ determining $\boldsymbol{G}_{\mathrm{H}}$ and $\boldsymbol{\Gamma}_{\mathrm{H}}$. All field relations are solved in dimensionless form. The effective shear modulus $\mu_{\mathrm{fcc}}$ is used as the basic energy density scaling. Together with the (constant isothermal) mobility $m_{0}$ from (6), this determines the time scaling $1 /\left(\mu_{\mathrm{fcc}} m_{0}\right)$. Length scaling is based on $d_{111}$, which also represents the grid point spacing in the numerical simulations. In particular, in this case, the dislocation core is resolved over multiple grid points in the slip plane (see for example Figure 4 in the text).

\section{B. Thermodynamic driving \& Peach-Köhler forces}

The purpose of this appendix is the relation of the evolution of the dislocation thermodynamic state and the related driving force inherent in the Ginzburg-Landau relation (6) to the Peach-Köhler force (PKF) and the results in Figure 6. To this end, consider a plane $S$ in the single crystal across which the crystal lattice has been slipped by dislocations. Let $S_{-}$represent the unslipped side, and $S_{+}$the slipped side, of $S$. Assume that the unit normal $\boldsymbol{n}_{S}$ to $S$ points from $S_{-}$to $S_{+}$. Let $\llbracket f \rrbracket_{S}:=f_{+}-f_{-}$represent the jump in the limits $f_{ \pm}$of any field $f$ on $S_{ \pm}$across $S$. In particular, for Volterra dislocations (e.g., Landau and Lifshitz, 1970; Hirth and Lothe, 1982; Teodosiu, 1982; Mura, 1987; Li and Wang, 2008; Balluffi, 2012), we have $\llbracket \boldsymbol{x} \rrbracket_{S}=\mathbf{0}$ and $\llbracket \boldsymbol{u} \rrbracket_{S} \neq \mathbf{0}$. In addition,

$$
\llbracket \nabla \boldsymbol{u} \rrbracket_{S}=\frac{\llbracket \boldsymbol{u} \rrbracket_{S}}{d_{S}} \otimes \boldsymbol{n}_{S}
$$

then holds for the distortion field across $S$ via the Hadamard lemma (e.g., Šilhavý, 1997, Proposition 2.1.6), $d_{S}$ being the "width" or "thickness" of $S$. Consider next the relation between $\llbracket \boldsymbol{u} \rrbracket_{S}$ 


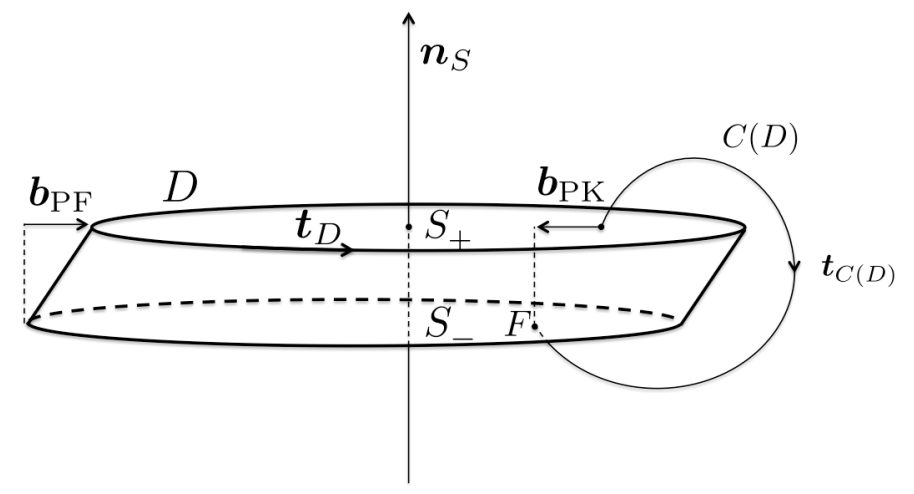

Figure B.1: Relation between the Burgers vector convention $\boldsymbol{b}_{\mathrm{PF}} \equiv \llbracket \boldsymbol{u} \rrbracket_{S}$ inherent in the residual distortion model (3)-(4) and that $\boldsymbol{b}_{\mathrm{PK}} \equiv-\llbracket \boldsymbol{u} \rrbracket_{S}$ (SF/RH convention) in the definition of PeachKöhler force. See text for details.

and the closure failure around a Burgers circuit. To this end, let $D$ represent a "right-handed" dislocation loop on $S$ as shown in Figure B.1. "Right-handed" here means that, when the unit tangent $t_{D}$ to $D$ is oriented in the direction of the (fingers of the) right hand, the right-hand thumb is parallel to $\boldsymbol{n}_{S}$, and $\boldsymbol{t}_{D} \cdot \boldsymbol{n}_{S}=0$. Let $C(D)$ represent a Burgers circuit around $D$ with unit tangent $\boldsymbol{t}_{C(D)}$ (Figure B.1) enclosing a surface $S(C)$ with unit normal $\boldsymbol{n}_{S(C)}$ in a right-handed fashion. In this case, at the point where $D$ intersects $S(C), \boldsymbol{t}_{D}$ is parallel to $\boldsymbol{n}_{S(C)}$. In this case, $C(D)$ starts on the slipped side $\boldsymbol{x}_{+} \in S_{+}$of $S$ and ends on the unslipped side $\boldsymbol{x}_{-} \in S_{-}$of $S$, and

$$
\oint_{C(D)} \nabla_{t_{C(D)}} \boldsymbol{u} d l=\boldsymbol{u}_{-}-\boldsymbol{u}_{+}=-\llbracket \boldsymbol{u} \rrbracket_{S}
$$

follows. This corresponds to the concept of "local" Burgers vector and the so-called SF/RH convention ("start-finish / right-hand"; e.g., Hirth and Lothe, 1982; Balluffi, 2012, Chapter 12). In particular, as shown in Figure B.1, $C(D)$ starts on $S_{+}$("start" in "SF") and ends on $S_{-}$at $F$ ("finish" in "SF").

Next, let $S=S_{a}$ represent any $\{111\}$ slip plane in the single crystal. Let $\boldsymbol{t}_{D a}$ be the unit tangent field of a (curved) dislocation line on $S_{a}$, and $\boldsymbol{m}_{D a}:=\boldsymbol{t}_{D a} \times \boldsymbol{n}_{a}$ the unit normal to $D$ tangent to $S_{a}$ in the direction of line bow-out (note $\boldsymbol{t}_{D a} \cdot \boldsymbol{n}_{a}=0$ ). Mechanical equilibrium $\llbracket \boldsymbol{T} \rrbracket_{a} \boldsymbol{n}_{a}=\mathbf{0}$ on $S_{a}$ then results in the form

$$
\llbracket \boldsymbol{T} \boldsymbol{n}_{a} \cdot \boldsymbol{u} \rrbracket_{a}=\boldsymbol{T}^{\mathrm{T}} \llbracket \boldsymbol{u} \rrbracket_{a} \cdot \boldsymbol{n}_{a}=\boldsymbol{T}^{\mathrm{T}} \boldsymbol{b}_{\mathrm{PK} a} \times \boldsymbol{t}_{D a} \cdot \boldsymbol{m}_{D a}
$$

for the component of the mechanical force $\boldsymbol{T}^{\mathrm{T}} \boldsymbol{b}_{\mathrm{PK} a} \times \boldsymbol{t}_{D a}$ per unit length in the direction $\boldsymbol{m}_{D a}$ on the dislocation with Burgers vector $\boldsymbol{b}_{\mathrm{PK} a}:=-\llbracket \boldsymbol{u} \rrbracket_{a}$ (see Figure B.1), i.e., of the PKF (e.g., Hirth and Lothe, 1982, Equation (4-43)). As usual, the PKF splits

$$
\boldsymbol{T}^{\mathrm{T}} \boldsymbol{b}_{\mathrm{PK} a} \times \boldsymbol{t}_{D a}=\left(\boldsymbol{b}_{\mathrm{PK} a} \cdot \boldsymbol{T} \boldsymbol{m}_{D a}\right) \boldsymbol{n}_{a}-\left(\boldsymbol{b}_{\mathrm{PK} a} \cdot \boldsymbol{T} \boldsymbol{n}_{a}\right) \boldsymbol{m}_{D a}
$$

into glide $-\boldsymbol{b}_{\mathrm{PK} a} \cdot \boldsymbol{T} \boldsymbol{n}_{a}$ and climb $\boldsymbol{b}_{\mathrm{PK} a} \cdot \boldsymbol{T} \boldsymbol{m}_{D a}$ components. Related to the former is the expression

$$
\boldsymbol{T} \boldsymbol{n}_{a} \cdot \llbracket \nabla \boldsymbol{u} \rrbracket_{a} \boldsymbol{n}_{a}=\left(\llbracket \boldsymbol{u} \rrbracket_{a} \cdot \boldsymbol{T} \boldsymbol{n}_{a}\right) / d_{a}=\left(\boldsymbol{b}_{\mathrm{PF} a} \cdot \boldsymbol{T} \boldsymbol{n}_{a}\right) / d_{a}
$$


obtained from (B.1) and mechanical equilibrium on $S_{a}$ for the mechanical work per unit volume performed by $\boldsymbol{T}$ in slipping $S_{a}$, with $\boldsymbol{b}_{\mathrm{PF} a}:=\llbracket \boldsymbol{u} \rrbracket_{a}$ (see Figure B.1). In turn, (B.5) is comparable to the elastic part

$$
-\partial_{\phi_{a}} \psi_{\mathrm{E}}=\boldsymbol{T} \cdot \boldsymbol{H}_{a}=\left(\boldsymbol{b}_{a} \cdot \boldsymbol{T} \boldsymbol{n}_{a}\right) / d_{a}=\left(\boldsymbol{b}_{\mathrm{PF} a} \cdot \boldsymbol{T} \boldsymbol{n}_{a}\right) / d_{a}
$$

via (1)-(4) of the "local" part $-\partial_{\phi_{a}} \psi$ of the non-local thermodynamic driving force $-\delta_{\phi_{a}} \psi$ determining the evolution of $\phi_{a}$ in (6). Consequently, the thermodynamic driving force $-\partial_{\phi_{a}} \psi_{\mathrm{E}}$ is comparable to the glide component $-\boldsymbol{b}_{\mathrm{PK} a} \cdot \boldsymbol{T} \boldsymbol{n}_{a}$ of the mechanical PKF. These are used to obtain the results for the PKF in Figure 6 in the text. 